\title{
On Riemann problems and front tracking for a model of sedimentation of polydisperse suspensions
}

\author{
Stefan Berres ${ }^{1}$ and Raimund Bürger ${ }^{1, *}$ \\ 1 Departamento de Ingeniería Matemática, Facultad de Ciencias Físicas y Matemáticas, Universidad de Concepción, \\ Casilla 160-C, Concepción, Chile
}

Received 24 February 2007, revised 30 July 2007, accepted 27 August 2007

Published online 18 October 2007

Key words Conservation law, Riemann problem, Liu entropy condition, front tracking, polydisperse suspensions. MSC (2000) 35L65, 76M25, 76T20

\begin{abstract}
This paper analyses a continuum model of the sedimentation of polydisperse suspensions, where the volume concentrations of the solids species are the sought unknowns. This leads to systems of conservation laws which are non-genuinely nonlinear ("non-convex") in the sense that they are only piecewise genuinely nonlinear. The solution of a Riemann problem is represented as a concatenation of elementary waves, where the linear degeneracy requires using the Liu entropy condition. After a general description of the composition of elementary waves, an algorithm for the computational construction is given. The solution of the Riemann problem is then utilized as a problem-adapted building block of a front tracking method. For the model of bidisperse suspensions, Riemann problems are classified by the location of the left and right state in the phase space of unknown variables. The front tracking method is applied to solve the initial value problem of a first-order hyperbolic $2 \times 2$ system of conservation laws describing the settling of a bidisperse suspension. The solution obtained by front tracking is compared with results obtained by an experiment and a finite difference scheme.
\end{abstract}

(C) 2007 WILEY-VCH Verlag GmbH \& Co. KGaA, Weinheim

\section{Introduction}

\subsection{Scope}

We consider a model of sedimentation of a polydisperse suspension with spherical solid particles belonging to $N$ species that differ in size or density [5]. This model leads to an initial value problem for a nonlinear system of conservation laws, which describes the evolution of solids concentrations as a function of depth $x$ and time $t$ :

$$
u_{t}+f(u)_{x}=0, \quad u \in \mathcal{D} \subseteq \mathbb{R}^{N}, \quad x \in \mathbb{R}, \quad t>0 ; \quad f \in\left(C^{3}(\mathcal{D})\right)^{N} .
$$

We recall that the system (1.1) is called strictly hyperbolic if, for every $u \in \mathcal{D}$, the Jacobian $\mathcal{J}_{f}(u)$ has $N$ distinct real eigenvalues (or characteristic speeds) $\lambda_{1}(u)<\cdots<\lambda_{N}(u)$, and consequently, $N$ linearly independent right eigenvectors $r_{1}(u), \ldots, r_{N}(u)$. Here, $\mathcal{D}$ is the phase space, whose elements are states. In the context of the sedimentation model, $\mathcal{D}$ is the bounded space of concentration vectors. The $i$-th characteristic field $\left(\lambda_{i}, r_{i}\right)$ is said to be genuinely nonlinear at $u \in \mathcal{D}$ if $r_{i}(u) \cdot \nabla \lambda_{i}(u) \neq 0$, and otherwise linearly degenerate at that state. Due to the nonlinearity of the flux vector $f$, the characteristic fields appearing in the sedimentation model are, however, neither genuinely nonlinear nor linearly degenerate for all $u \in \mathcal{D}$, but only piecewise genuinely nonlinear. We refer to such systems as non-genuinely nonlinear or non-convex. Solutions of (1.1) are in general discontinuous, and besides the standard Rankine-Hugoniot jump condition, one needs to impose an additional entropy condition to select admissible discontinuities. Due to the nonconvexity of the system, the proper entropy condition for piecewise smooth solutions is the Liu entropy condition, which generalizes the well-known Oleŭnik entropy condition for the scalar case.

In this paper, we first recall the construction of solutions of the Riemann problem, which consists of the non-genuinely nonlinear system (1.1) along with the initial datum

$$
u(0, x)=u_{0}(x)= \begin{cases}u_{\mathrm{L}} & \text { for } x<0, \\ u_{\mathrm{R}} & \text { for } x>0 .\end{cases}
$$

\footnotetext{
* Corresponding author, e-mail: rburger@ ing-mat.udec.cl, Phone: +5641 2203126, Fax: +5641 2522055
} 
These solutions are constructed such that they satisfy the Liu entropy condition. We then implement the Riemann solver within a front tracking algorithm, which is applied to the problem of sedimentation of an initially homogeneous suspension, which is defined by two adjacent Riemann problems. While the solution strategy of the Riemann problem and the front tracking algorithm are presented for general $N$, for the numerical example, which is based on experimental data by Schneider et al. [47], we choose $N=2$. While substantial advances were made in recent years in the global hyperbolicity (type) analysis $[5,11]$ and in the design of high-resolution finite volume schemes for the sedimentation model (and related multi-species kinematic models) $[10,12,52]$, the present paper contributes to providing insight into the structure of exact solutions of the model and a building block for the hyperfast front tracking method [22].

The Riemann solver concatenates elementary waves, where we recall that an elementary wave is an entropy solution of a Riemann problem associated to one single characteristic field. A wave is a sequence of elementary waves, which is a piecewise continuous solution to a Riemann problem which assumes values in $\mathcal{D}$, and can be represented as a profile, a curve in phase space, or by an $x$-t-diagram. For each characteristic family, an elementary curve is a set of states in $\mathcal{D}$ that can be connected to a given state $u_{\mathrm{L}}$ by an elementary wave that satisfies the Liu entropy condition. An elementary curve consists of segments of branches of Hugoniot loci, integral curves and mixed curves. We parametrize elementary waves by arc lengths of the underlying elementary curves in $\mathcal{D}$, which allows us to solve a Riemann problem as a zero problem, where the projected right state is fitted to the prescribed right state.

\subsection{Related work}

The polydisperse sedimentation model goes back to Masliyah [41] and Lockett and Bassoon [38] ("MLB model"); further details are given in $[4,5,11,53]$. This model is similar to other multi-species kinematic models, for example for traffic flow [3,12,51], granular flow [19], and the settling of oil-in-water emulsions [46]. The settling of monodisperse suspensions, which is described by a scalar equation, is extensively treated in [14]. In particular, exact entropy solutions of the Riemann problem are constructed.

While the ultimate existence result for non-genuinely nonlinear systems with no further assumption than strict hyperbolicity is given by Bianchini [6] and Bianchini and Bressan [7], we focus in this discussion on specific solutions of the Riemann problem for a system of conservation laws corresponding to several particular applications. Fried and Roy [19] solve the Riemann problem for a model of granular flow by stating an explicit formula for both Hugoniot loci and rarefaction curves. This formula is feasible since they consider a sub-case of a more general model; in this sub-case, the system is genuinely nonlinear. For our model, which is not genuinely nonlinear, an analytical solution is not available. We utilize here a semi-analytical approach, by which we understand an analytical solution that involves the numerical solution of ordinary differential equations and non-linear systems of equations. Semi-analytical methods have been applied to systems of conservation laws in various applications in numerous works including [1,27,28,40,42,43,49]. In [27], a general algorithm for the solution of Riemann problems is proposed and applied to three-phase flow in porous media (see also [28]). However, the algorithm is restricted to $2 \times 2$ systems and general flux functions with one single manifold of linear degeneracy per characteristic family. The predictor-corrector algorithm of [27] is applied to a specific case of a rarefaction-shock combination. Also, for the solution of a Riemann problem, elementary waves are connected by the intersection of the 1-wave starting at the left state with the 2-wave starting at the right state, rather than by the optimization of wave lengths, where one starts in the left state and fits the concatenated elementary waves to the prescribed right state. In [1], two sets of Euler equations describe two-phase reactive granular materials. The system is non-strictly hyperbolic and non-conservative. The construction is based on the work of [42] for one Euler equation with non-convex flux functions. There, more general Cauchy problems are not solved, which could be done by using the Riemann solver presented in this paper along with a front tracking algorithm. In [49], where MHD equations are studied, wave lengths are found by solving a system of equations. Another non-genuinely nonlinear system are the Euler equations with non-convex flux function. For these equations, Müller and Voß [43] construct an elementary curve, from which an elementary wave is extracted.

In the present work, the front tracking method is applied as a computational tool to tackle an initial value problem. This numerical approach complements a theoretical viewpoint, where "wave" front tracking is seen as a further stage of the Glimm scheme, leading to a stability result that gives uniqueness of the solution (in addition to its existence), and its continuous dependence on the initial data (see [8] for an overview). In fact, historically, numerical applications such as polymer flooding [45] and chromatography [50] preceded the development of the more theoretical perspective. More recently, the front tracking method was also coupled with a finite difference scheme for polymer flooding [20] and combined with streamline simulations for the solution of three-dimensional problems of three-phase and multicomponent flows through porous media [25, 26, 35], where numerical solutions for both 1D and 3D problems are compared. The numerical solution of a specific application by front tracking always requires structural knowledge on the specific system. For instance, an exact Riemann solution valid for any left and right state is available for certain models of flow through porous media and of chromatography with Langmuir isotherms. In the latter case, the systems are Temple systems [50]. 


\subsection{This paper}

This paper proposes a strategy for the solution of specific initial value problems using the front tracking framework and a Riemann solver as a building block. The solution of the Riemann problem decisively depends on the shape of the integral curves (of the eigenvector field of the Jacobian of the flux function) in the phase space (hodograph plane). Thus, the Riemann solver eventually needs to be adjusted for a particular case by providing a good initial guess of the length and direction of the elementary curves. It is not applicable as soon as transitional waves or detached shocks appear, which in particular may occur for non-strictly hyperbolic systems (see [17, 18,35] for observations of this phenomenon in threephase flow in porous media). However, it is demonstrated that the proposed solution strategy can be successfully applied to strictly hyperbolic systems without detached Hugoniot loci even in the absence of knowledge of exact Riemann solutions, which is typical for polydisperse suspensions.

The remainder of this paper is organized as follows. In Sect. 2, we introduce the sedimentation model, which gives rise to an initial value problem for a non-genuinely nonlinear system of conservation laws of the form (1.1), where the unknowns are the concentrations of each species as a function of depth and time. For particles having the same density (i.e., that differ in size only), the system is strictly hyperbolic [5].

In Sect. 3 we establish a notational framework for the description of elementary waves and elementary curves, which are associated with one characteristic family only, in order to motivate the computational construction in Sect. 4. Briefly, the construction of elementary curves proceeds as follows. First, a state $u_{\mathrm{L}}$, sometimes referred to as anchor state, a characteristic family and a construction direction are chosen. Then, segments of branches of the Hugoniot locus, rarefaction curves and mixed curves are iteratively appended to an already constructed part of the elementary curve. The lengths of the appended segments are determined by the Liu entropy condition. The basic concepts of integral curves (Sect. 3.1), Hugoniot loci (Sect. 3.2) and the Liu entropy condition (Sect. 3.3) are recalled. Then, genuinely nonlinear systems and systems whose characteristic fields are either genuinely nonlinear or linearly degenerate (Sect. 3.4) are considered. To demonstrate the construction of elementary curves in the phase space of unknowns, first, a general formula for elementary curves with one (Sect. 3.5), and then, with a finite number of manifolds of linear degeneracy (Sect. 3.6) is given. Special focus is put on the parametrization, where jumps are parametrized by their jump strength and integral curves by arc length. Finally, in Sect. 3.7 different curve segment types in an elementary curve are characterized in order to specify the possible transitions between segments of different types.

In Sect. 4, the algorithm for the semi-analytic solution of Riemann problems is described. The general solution strategy for the front tracking algorithm requires a problem-adapted Riemann solver, which in turn concatenates elementary waves. Thus, the solution of a Riemann problem is represented as concatenation of elementary waves with fitted curve length. For the construction of elementary waves, the Liu entropy condition has to be obeyed.

In Sect. 5, it is shown how the global sedimentation behavior of a bidisperse suspension can be deduced from algebraic conditions on a characteristic family in the phase space, which generalizes the geometric inspection of the flux plot for scalar equations. In Sect. 5.1, the well-known construction of global solutions for scalar equations in the theory of sedimentation is briefly reviewed. In Sect. 5.2 the concept of a free Riemann problem is introduced. In Sect. 5.3, a test example (Example 2) of a bidisperse suspension is introduced. This example is based on the analysis and experiment by Schneider et al. [47]. It is demonstrated that the Liu entropy condition is violated if it is assumed that the solution of all initial Riemann problems consists of jumps only. In Sects. 5.4-5.7, this test example is solved by the front tracking method [22], which generalizes the polygonal approximation employed for scalar equations [13, 15,21]: In Sect. 5.4 regions in the phase space of volume concentrations are identified that correspond to different modes of sedimentation. In Sect. 5.5 the application of the front tracking method to the test example is reported, which builds on the classification of Riemann problems (Sect. 5.7). The structure of the global solution is described in Sect. 5.8. Finally, in Sect. 5.9, the front tracking solution is compared with the result of a finite difference scheme.

\section{Model of sedimentation of polydisperse suspensions}

We assume that the mixture consists of particles of $N$ different species suspended in a fluid, where the particles have diameters $d_{1} \geq d_{2} \geq \cdots \geq d_{N}$ and densities $\varrho_{1}, \ldots, \varrho_{N}$, where $d_{i} \neq d_{j}$ or $\varrho_{i} \neq \varrho_{j}$ for $i \neq j$. According to the MLB model, the evolution of the volume fractions $\phi_{1}, \ldots, \phi_{N}$ of all species as a function of depth $z$ and time $t$ is given by the system of conservation laws

$$
\partial_{t} \phi_{i}+\partial_{z} f_{i}(\Phi)=0, \quad i=1, \ldots, N
$$

together with an initial concentration distribution and zero-flux boundary conditions:

$$
\Phi(z, 0)=\Phi^{0}(z), \quad 0 \leq z \leq L
$$




$$
f_{i}(\Phi)=0 \quad \text { for } z=0 \text { and } z=L, \quad i=1, \ldots, N .
$$

The flux density vector $f(\Phi):=\left(f_{1}(\Phi), \ldots, f_{N}(\Phi)\right)^{\mathrm{T}}$ models the hindrance of the movement of each species through the presence of others combined with segregation driven by fluctuations of the volume density of the mixture. According to the MLB model, the components of $f(\Phi)$ are given by

$$
f_{i}(\Phi)=f_{i}^{\mathrm{M}}(\Phi):=\chi_{\mathcal{D}}(\Phi) \mu \phi_{i}\left[\delta_{i}\left(\bar{\varrho}_{i}-\bar{\varrho}^{\mathrm{T}} \Phi\right)-\sum_{k=1}^{N} \delta_{k} \phi_{k}\left(\bar{\varrho}_{k}-\bar{\varrho}^{\mathrm{T}} \Phi\right)\right] V(\phi), \quad i=1, \ldots, N,
$$

where $\mu=g d_{1}^{2} /\left(18 \mu_{\mathrm{f}}\right)$ is a viscosity parameter, $g$ is the acceleration of gravity and $\mu_{\mathrm{f}}$ is the viscosity of the fluid, $\delta_{i}:=d_{i}^{2} / d_{1}^{2}, \bar{\varrho}_{i}:=\varrho_{i}-\varrho_{\mathrm{f}}, i=1, \ldots, N$, where $\varrho_{\mathrm{f}}$ is the fluid density, and $\bar{\varrho}:=\left(\bar{\varrho}_{1}, \ldots, \bar{\varrho}_{N}\right)^{\mathrm{T}}$. The function $V(\phi)$ is a hindered settling factor, which can be given by

$$
V(\phi)=\left\{\begin{array}{ll}
(1-\phi)^{C-2} & \text { for } 0 \leq \phi<1, \\
0 & \text { otherwise }
\end{array} \quad C>2, \quad \phi:=\phi_{1}+\cdots+\phi_{N} .\right.
$$

A typical value for real suspensions is $C=4.7$ [47], and we assume that $V(\phi)>0$ and $V^{\prime}(\phi)<0$ for $0<\phi<1$. A non-standard feature of the sedimentation model is that the flux is discontinuously set to zero by the factor $\chi_{\mathcal{D}}(\Phi)$, such that $f(\Phi)=0$ for $\Phi \notin \mathcal{D}$, where $\chi_{\mathcal{D}}$ is the characteristic function of the domain of relevant concentration values

$$
\mathcal{D}:=\left\{\Phi \in \mathbb{R}^{N}: \phi_{1} \geq 0, \ldots, \phi_{N} \geq 0, \phi_{1}+\cdots+\phi_{N} \leq \phi^{\infty}(\Phi)\right\}
$$

which is bounded by an implicitly defined curve in phase space, the so-called maximum packing manifold

$$
\partial^{\infty}:=\left\{\Phi: \phi_{1}+\cdots+\phi_{N}=\phi^{\infty}(\Phi)\right\},
$$

which describes the composition of a packed polydisperse sediment. An example for $N=2$ is due to Jeschar (cited in [47]), where $\phi_{\max }=0.68$ is the packing density of equal-sized spheres:

$$
\phi^{\infty}=\left(1-\phi_{\max }\right)\left(1-\delta_{2}^{D}\right) \phi_{1}+\phi_{\max }, \quad D=0.3 \sin \left(\pi \phi_{2} / \phi\right), \quad \phi_{1} \in\left[0, \phi_{\max }\right] .
$$

Note that for $N=2, \delta_{2}<1$ and $\phi_{1}>0$, we have that $\phi^{\infty}>\phi_{\max }$, which reflects that smaller particles may occupy interstices between larger particles. Since the flux functions (2.4) are cut at the boundary of $\mathcal{D}$, we may replace (2.2) and (2.3) by the following initial condition, where $\Phi_{\infty} \in \partial^{\infty}$ may either be prescribed or is part of the solution:

$$
\Phi(z, 0)= \begin{cases}0 & \text { for } z<0 \\ \Phi_{0} & \text { for } 0 \leq z<L, \\ \Phi_{\infty} & \text { for } z \geq L .\end{cases}
$$

For equal-density particles, we define $\bar{\varrho}_{\mathrm{s}}:=\bar{\varrho}_{1}=\cdots=\bar{\varrho}_{N}$ and $\boldsymbol{\delta}:=\left(\delta_{1}, \ldots, \delta_{N}\right)^{\mathrm{T}}$. Then (2.4) simplifies to

$$
f_{i}^{\mathrm{M}}(\Phi)=\chi_{\mathcal{D}}(\Phi) \mu(1-\phi) \bar{\varrho}_{\mathrm{s}} \phi_{i}\left(\delta_{i}-\delta^{\mathrm{T}} \Phi\right) V(\phi), \quad i=1, \ldots, N,
$$

and we have the following theorem proved in [5] (see also [11] for the case $N=2$ ).

Theorem 2.1. If $\varrho_{1}=\cdots=\varrho_{N}=\varrho_{\mathrm{s}}, \delta_{1}>\delta_{2}>\cdots>\delta_{N}$, and $\Phi \in \mathcal{D}^{0}$, then (2.1) is strictly hyperbolic. Precisely, if we define $\gamma^{\infty}:=-2 \boldsymbol{\delta}^{\mathrm{T}} \Phi V(\phi)(1-\phi)+(V(\phi)(1-\phi))^{\prime}\left(\boldsymbol{\delta}^{\mathrm{T}} \Phi+\phi\right)$, then the eigenvalues $\nu_{1}(\Phi), \ldots, \nu_{N}(\Phi)$ of $\mathcal{J}_{f}(\Phi)$ satisfy

$$
\begin{aligned}
& \nu_{i}(\Phi) \in\left(\mu \bar{\varrho}_{\mathrm{s}} V(\phi)(1-\phi)\left(\delta_{i}-\delta^{\mathrm{T}} \Phi\right), \mu \bar{\varrho}_{\mathrm{s}} V(\phi)(1-\phi)\left(\delta_{i+1}-\delta^{\mathrm{T}} \Phi\right)\right), \quad i=1, \ldots, N-1, \\
& \nu_{N}(\Phi) \in\left(\mu \bar{\varrho}_{\mathrm{s}} V(\phi)(1-\phi)\left(\delta_{N}-\delta^{\mathrm{T}} \Phi\right), \mu \bar{\varrho}_{\mathrm{s}} \gamma^{\infty}\right) .
\end{aligned}
$$

\section{The Riemann problem for a system of conservation laws}

\subsection{Integral curves and rarefaction waves}

The $i$-rarefaction curve (integral curve) is the solution of the initial value problem for a system of ordinary differential equations

$$
\frac{d \mathcal{R}_{i}\left(u_{\mathrm{L}}, \zeta_{i}\right)}{d \zeta_{i}}=r_{i}\left(\mathcal{R}_{i}\left(u_{\mathrm{L}}, \zeta_{i}\right)\right), \quad \zeta_{i}>0, \quad \mathcal{R}_{i}\left(u_{\mathrm{L}}, \zeta_{i}=0\right)=u_{\mathrm{L}}
$$


where $r_{i}$ is assumed to point in the direction of increasing $\lambda_{i}$. We recall that when $\zeta_{i} \mapsto \lambda_{i}\left(\mathcal{R}_{i}\left(u_{\mathrm{L}}, \zeta_{i}\right)\right)$ is increasing and $u_{\mathrm{R}}=\mathcal{R}_{i}\left(u_{\mathrm{L}}, \zeta_{\mathrm{R}}\right)$ for some $\zeta_{\mathrm{R}}>0$, then the Riemann problem (1.1), (1.2) is solved by the $i$-rarefaction wave

$$
u(x, t)= \begin{cases}u_{\mathrm{L}} & \text { for } x / t \leq \lambda_{i}\left(u_{\mathrm{L}}\right), \\ w_{i}(x / t) & \text { for } \lambda_{i}\left(u_{\mathrm{L}}\right)<x / t<\lambda_{i}\left(u_{\mathrm{R}}\right), \\ u_{\mathrm{R}} & \text { for } \lambda_{i}\left(u_{\mathrm{R}}\right) \leq x / t\end{cases}
$$

where $w_{i}$ is a solution of the system of ordinary differential equations

$$
\mathcal{J}_{f}\left(w_{i}(\xi)\right) w_{i}^{\prime}(\xi)=\xi w_{i}^{\prime}(\xi), \quad \xi:=x / t, \quad i=1, \ldots, N,
$$

which has either the trivial solution $w_{i}^{\prime}(\xi) \equiv 0$, leading to constant $w_{i}$, or $w_{i}(\xi)$ is the solution of

$$
w_{i}^{\prime}(\xi)=r_{i}\left(w_{i}(\xi)\right)\left(r_{i}\left(w_{i}(\xi)\right) \cdot \nabla \lambda_{i}\left(w_{i}(\xi)\right)\right)^{-1}, \quad w_{i}\left(\xi_{\mathrm{L}}\right)=u_{\mathrm{L}}, \quad \xi \in\left[\xi_{\mathrm{L}}=\lambda_{i}\left(u_{\mathrm{L}}\right), \xi_{\mathrm{R}}=\lambda_{i}\left(u_{\mathrm{R}}\right)\right] .
$$

\subsection{Hugoniot loci and shocks}

Jumps in the solution between two states $u^{-}, u^{+} \in \mathbb{R}^{N}$ need to satisfy the well-known Rankine-Hugoniot condition

$$
f\left(u^{-}\right)-f\left(u^{+}\right)=\sigma\left(u^{-}, u^{+}\right)\left(u^{-}-u^{+}\right),
$$

where $\sigma\left(u^{-}, u^{+}\right) \in \mathbb{R}$ is the jump propagation speed. The Hugoniot locus $\mathcal{H}\left(u_{\mathrm{L}}\right)$ associated with a state $u_{\mathrm{L}}$ is the set

$$
\mathcal{H}\left(u_{\mathrm{L}}\right):=\left\{u \in \mathcal{D}: \exists \sigma=\sigma\left(u_{\mathrm{L}}, u\right) \in \mathbb{R}: f\left(u_{\mathrm{L}}\right)-f(u)=\sigma\left(u_{\mathrm{L}}-u\right)\right\}
$$

of all states $u$ for which the pair $\left(u_{\mathrm{L}}=u^{-}, u=u^{+}\right)$satisfies (3.4). If (1.1) is strictly hyperbolic in $u_{\mathrm{L}}$, then $\mathcal{H}\left(u_{\mathrm{L}}\right)$ consists locally of the union of $N$ branches $\mathcal{H}_{1}\left(u_{\mathrm{L}}\right), \ldots, \mathcal{H}_{N}\left(u_{\mathrm{L}}\right)$. (For highly non-linear systems, especially for nonstrictly hyperbolic systems (not in our case), detached branches of the Hugoniot locus may occur [35]; these features are, however, not observed in the present class of models.) Each branch can be represented as a function $\mathcal{H}_{i}:\left(u_{\mathrm{L}}, \zeta_{i}\right) \mapsto u$ satisfying

$$
f\left(\mathcal{H}_{i}\left(u_{\mathrm{L}}, \zeta_{i}\right)\right)-f\left(u_{\mathrm{L}}\right)=\sigma\left(\mathcal{H}_{i}\left(u_{\mathrm{L}}, \zeta_{i}\right), u_{\mathrm{L}}\right)\left(\mathcal{H}_{i}\left(u_{\mathrm{L}}, \zeta_{i}\right)-u_{\mathrm{L}}\right), \quad \mathcal{H}_{i}\left(u_{\mathrm{L}}, 0\right)=u_{\mathrm{L}}
$$

Here, $\zeta_{i}$ parametrizes the shock strength, which is a distance in phase space, as

$$
\left|\zeta_{i}\right|=\left\|u_{\mathrm{L}}-\mathcal{H}_{i}\left(u_{\mathrm{L}}, \zeta_{i}\right)\right\|_{2}
$$

and is directed such that $d \mathcal{H}_{i}\left(u_{\mathrm{L}}, \zeta_{i}\right) /\left.d \zeta_{i}\right|_{\zeta_{i}=0}=r_{i}\left(u_{\mathrm{L}}\right)$. The parameters $\zeta_{i}$ are signed such that $\zeta_{i}>0$ on those parts of $\mathcal{H}_{i}$ where $\sigma\left(u_{\mathrm{L}}, u\right) \leq \lambda_{i}\left(u_{\mathrm{L}}\right)$ for any $u \in \mathcal{H}_{i}\left(u_{\mathrm{L}}\right):=\mathcal{H}_{i}\left(u_{\mathrm{L}}, \cdot\right)$ with $\left\|u-u_{\mathrm{L}}\right\|_{2}$ sufficiently small.

\subsection{The Liu entropy condition}

An $i$-shock between $u_{\mathrm{L}}$ and $u_{\mathrm{R}} \in \mathcal{H}_{i}\left(u_{\mathrm{L}}\right)$ is said to satisfy the Liu entropy condition if

$$
\sigma\left(u_{\mathrm{L}}, u_{\mathrm{R}}\right) \leq \sigma\left(u_{\mathrm{L}}, u\right) \quad \text { for all } u \in \mathcal{H}_{i}\left(u_{\mathrm{L}}\right)
$$

This condition, which generalizes both the Oleŭnik entropy condition for scalar equations and the Lax entropy condition for genuinely nonlinear (convex) systems, is equivalent to the vanishing viscosity characterization if the viscosity matrix is the identity matrix multiplied by a viscosity parameter [37,39].

We wish to describe characteristic fields which are globally neither genuinely nonlinear, nor linearly degenerate, and may exhibit general areas of linear degeneracy in phase space, so-called manifolds of linear degeneracy (MLDs)

$$
\mathcal{M}_{i}:=\left\{u \in \mathcal{D}: r_{i}(u) \cdot \nabla \lambda_{i}(u)=0\right\}
$$

which are assigned to the $i$-th characteristic family. For the closed-form representation of the elementary curve in Sects. 3.5 and 3.6, the MLDs are assumed to be of codimension one (that is, curves for $2 \times 2$ systems, surfaces for $3 \times 3$ systems). This restriction is relaxed in Sect. 3.7, where an elementary curve is assembled as a sequence of curve segments. Such a general MLD (not restricted to be of codimension one) could be easily constructed in the model framework by letting the hindrance function $V(\phi)$ be constant on an interval. However, our example taken from literature provides a MLD of codimension one. 


\subsection{Genuinely nonlinear systems}

If all families $\left(\lambda_{i}, r_{i}\right)$ are genuinely nonlinear, then the system is called genuinely nonlinear. This paragraph summarizes the known construction of solutions for Riemann problems for such systems. To this end, in this section (1.1) is assumed to be genuinely nonlinear.

For $u_{\mathrm{R}} \in \mathcal{H}_{i}\left(u_{\mathrm{L}}\right)$, a piecewise constant discontinuous solution of (1.1), (1.2) is [34]

$$
u(x, t)=\left\{\begin{array}{ll}
u_{\mathrm{L}} & \text { for } x / t<\sigma\left(u_{\mathrm{L}}, u_{\mathrm{R}}\right), \\
u_{\mathrm{R}} & \text { for } x / t>\sigma\left(u_{\mathrm{L}}, u_{\mathrm{R}}\right),
\end{array} \quad \text { where } f\left(u_{\mathrm{L}}\right)-f\left(u_{\mathrm{R}}\right)=\sigma\left(u_{\mathrm{L}}, u_{\mathrm{R}}\right)\left(u_{\mathrm{L}}-u_{\mathrm{R}}\right),\right.
$$

while for $u_{\mathrm{R}} \in \mathcal{R}_{i}\left(u_{\mathrm{L}}\right)$ a continuous solution (rarefaction wave) is given by (3.2) with $\zeta_{i}=x / t$.

A necessary condition for (3.2) and (3.3) to solve the Riemann problem (1.1), (1.2) is that the eigenvalues of the $i$-th family $\xi=\lambda_{i}\left(w_{i}(\xi)\right)$ strictly increase along $\mathcal{R}_{i}\left(u_{\mathrm{L}}\right)$. In view of (3.1), the directional derivative or speed rate $r_{i}(u) \cdot \nabla \lambda_{i}(u)$ must be positive along that branch of the rarefaction curve $\mathcal{R}_{i}\left(u_{\mathrm{L}}\right)$ which can be used for the construction of the rarefaction wave solution. This relevant part of $\mathcal{R}_{i}\left(u_{\mathrm{L}}\right)$ is a rarefaction curve. The choice of $u_{\mathrm{L}}$ as left Riemann constant is essential, since $u_{\mathrm{L}}$ is left only in direction of increasing $|\xi|$.

Now fix $u_{\mathrm{R}}$ and select $u_{\mathrm{L}} \in \mathcal{H}\left(u_{\mathrm{R}}\right)$. Then the solution is given by (3.7) if the Lax entropy condition [32]

$$
\lambda_{i}\left(u_{\mathrm{L}}\right) \geq \sigma\left(u_{\mathrm{L}}, u_{\mathrm{R}}\right) \geq \lambda_{i}\left(u_{\mathrm{R}}\right)
$$

is satisfied, which ensures that $i$-characteristics disappear into the shock as time advances. To find all states $u_{\mathrm{L}} \in \mathcal{H}\left(u_{\mathrm{R}}\right)$ admitting a discontinuous solution (3.7) satisfying (3.8), that branch of $\mathcal{H}_{i}\left(u_{\mathrm{R}}\right)$ has to be followed which has second-order contact to that branch of $\mathcal{R}_{i}\left(u_{\mathrm{R}}\right)$ along which $\lambda_{i}$ decreases. These "entropy-satisfying" portions of Hugoniot curves are referred to as shock curves, denoted $\mathcal{S}_{i}\left(u_{\mathrm{R}}\right), i=1, \ldots, N$, each of which leaves the $u_{\mathrm{R}}$ in a direction opposite to that of the corresponding rarefaction curve $\mathcal{R}_{i}\left(u_{\mathrm{R}}\right)$.

For $N=2$, one can solve the Riemann problem for $u_{0}:=u_{\mathrm{L}}$ and $u_{2}=u_{\mathrm{R}}$ by determining an intermediate state $u_{1}$ by intersecting the wave curves $\mathcal{W}_{1}\left(u_{\mathrm{L}}\right):=\mathcal{R}_{1}\left(u_{\mathrm{L}}\right) \circ \mathcal{S}_{1}\left(u_{\mathrm{L}}\right)$ and $\mathcal{W}_{2}\left(u_{\mathrm{R}}\right):=\mathcal{R}_{2}\left(u_{\mathrm{R}}\right) \circ \mathcal{S}_{2}\left(u_{\mathrm{R}}\right)$. In fact, $\mathcal{W}_{1}\left(u_{\mathrm{L}}\right)$ is the locus of all "right" states that can be connected to $u_{\mathrm{L}}$ by an entropy-satisfying 1-wave (either a shock or a rarefaction wave), while $\mathcal{W}_{2}\left(u_{\mathrm{R}}\right)$ is the locus of all "left states" that can be connected to the right state $u_{\mathrm{R}}$ by an entropy-satisfying 2-wave. The determination of $u_{1}$ and thus the explicit solution $u=u(x, t)$ of the Riemann problem generally involves the solution of nonlinear systems of algebraic equations and ordinary differential equations. For general $N$ we need to determine $N-1$ intermediate states. This solution structure is also valid for systems with linearly degenerate characteristic fields (which, however, does not occur in the application under study). For such fields, no centered rarefaction waves exist, and the rarefaction curves coincide with the Hugoniot locus. This means that if the $i$-th characteristic field is linearly degenerate, then the states $u_{i-1}$ and $u_{i}$ occurring in the solution construction are separated by a contact discontinuity that satisfies (3.8) with $u_{\mathrm{L}}=u_{i-1}, u_{\mathrm{R}}=u_{i}$ and equalities holding. In contrast to this, shocks in genuinely nonlinear families, for which (3.8) holds with inequalities on both sides, are called compressive. This construction leads to a constructive existence and uniqueness proof for general $N$ provided that the curves $\mathcal{W}_{1}, \ldots, \mathcal{W}_{N}$ form a coordinate system on $\mathcal{D}$. This is certainly valid if $\left|u_{\mathrm{L}}-u_{\mathrm{R}}\right|$ is small. The following theorem is stated in [16]; see [32] for its proof.

Theorem 3.1. Assume that (1.1) is strictly hyperbolic and that each characteristic family is either genuinely nonlinear or linearly degenerate. If $\left|u_{\mathrm{R}}-u_{\mathrm{L}}\right|$ is sufficiently small, then there exists a unique solution $u=u(x / t)$ of the Riemann problem (1.1), (1.2) with small total variation, (which for $N=2$ comprises three constants $u_{0}=u_{\mathrm{L}}, u_{1}, u_{2}=u_{\mathrm{R}}$ ). When the $i$-th characteristic family is linearly degenerate, $u_{i}$ is joined to $u_{i-1}$ by a contact discontinuity, while when it is genuinely nonlinear, $u_{i}$ is joined to $u_{i-1}$ either by a rarefaction wave or a compressive shock.

\subsection{Characteristic fields with single linear degeneracy}

If the $i$-th characteristic field includes one single MLD $\mathcal{M}_{i}$ of codimension one, then the solution of the Riemann problem contains an elementary wave consisting of both a rarefaction wave and a contact discontinuity whenever $u_{\mathrm{L}}$ and $u_{\mathrm{R}}$ are located on different sides of $\mathcal{M}_{i}$, that is, $D_{r_{i}} \lambda_{i}\left(u_{\mathrm{L}}\right) \cdot D_{r_{i}} \lambda_{i}\left(u_{\mathrm{R}}\right)<0$. There are two possible cases [2], as follows.

Case 1. If $D_{r_{i}}^{2} \lambda_{i}\left(u_{\mathrm{L}}\right)>0$, then the elementary wave consists of a rarefaction wave followed by a left contact discontinuity. Given $u_{\mathrm{L}}$, there is a point $u^{\mathcal{R}_{i}} \in \mathcal{R}_{i}\left(u_{\mathrm{L}}, \zeta\right), \zeta>0$ such that $u_{\mathrm{R}} \in \mathcal{H}_{i}\left(u^{\mathcal{R}_{i}}\right)$ and $\lambda_{i}\left(u^{\mathcal{R}_{i}}\right)=\sigma\left(u^{\mathcal{R}_{i}}, u_{\mathrm{R}}\right)$. The curve is equipped with a signed parametrization such that

$$
\left.r_{i}\left(u_{\mathrm{L}}\right) \cdot \partial_{\zeta} \mathcal{R}_{i}\left(u_{\mathrm{L}}, \zeta\right)\right|_{\zeta=0}>0 \quad \text { and }\left.\quad r_{i}\left(u^{\mathcal{R}_{i}}\right) \cdot \partial_{\zeta} \mathcal{H}_{i}\left(u^{\mathcal{R}_{i}}, \zeta\right)\right|_{\zeta=0}>0
$$


If we introduce the parameters $\zeta^{\mathcal{C}_{i}}, u^{\mathcal{R}_{i}}(\zeta)$, and $u(\zeta)$ such that

$$
\begin{aligned}
& u^{\mathcal{R}_{i}}(\zeta)=\mathcal{R}_{i}\left(u_{\mathrm{L}}, \zeta^{\mathcal{C}_{i}}-\zeta\right), \quad u(\zeta) \in \mathcal{H}_{i}\left(u^{\mathcal{R}_{i}}(\zeta)\right), \\
& \lambda_{i}\left(u^{\mathcal{R}_{i}}(\zeta)\right)=\sigma\left(u^{\mathcal{R}_{i}}(\zeta), u(\zeta)\right), \quad\left(r_{i} \cdot \nabla \lambda_{i}\right)\left(\mathcal{R}_{i}\left(u_{\mathrm{L}}, \zeta^{\mathcal{C}_{i}}\right)\right)=0,
\end{aligned}
$$

then the resulting elementary curve is given by

$$
\mathcal{E}\left(u_{\mathrm{L}}, \zeta\right)= \begin{cases}\mathcal{R}_{i}\left(u_{\mathrm{L}}, \zeta\right) & \text { for } \zeta \in\left[0, \zeta^{\mathcal{C}_{i}}\right), \\ u(\zeta) & \text { for } \zeta \in\left[\zeta^{\mathcal{C}_{i}}, \zeta_{\mathrm{R}}\right]\end{cases}
$$

Case 2. If $D_{r_{i}}^{2} \lambda_{i}\left(u_{\mathrm{L}}\right)<0$, then the constructed elementary curve is a composite curve which consists of a right contact discontinuity followed by a rarefaction wave. If $u_{\mathrm{L}}$ is given, then there is some $u^{\mathcal{R}_{i}^{*}} \in \mathcal{H}_{i}\left(u_{\mathrm{L}}\right)$ such that $\sigma\left(u_{\mathrm{L}}, u^{\mathcal{R}_{i}^{*}}\right)=$ $\lambda_{i}\left(u^{\mathcal{R}_{i}^{*}}\right)$ and $u_{\mathrm{R}} \in \mathcal{R}_{i}\left(u^{\mathcal{R}_{i}^{*}}\right)$. The curve is then equipped with a signed parametrization such that

$$
\left.r_{i}\left(u_{\mathrm{L}}\right) \cdot \partial_{\zeta} \mathcal{H}_{i}\left(u_{\mathrm{L}}, \zeta\right)\right|_{\zeta=0}<0,\left.\quad r_{i}\left(u^{\mathcal{R}_{i}^{*}}\right) \cdot \partial_{\zeta} \mathcal{R}_{i}\left(u^{\mathcal{R}_{i}^{*}}, \zeta\right)\right|_{\zeta=0}>0
$$

The unknown parameters $\zeta^{\mathcal{R}_{i}^{*}}$ and $\zeta_{\mathrm{R}}$ satisfy

$$
u^{\mathcal{R}_{i}^{*}}=\mathcal{H}_{i}\left(u_{\mathrm{L}}, \zeta^{\mathcal{R}_{i}^{*}}\right), \quad u_{\mathrm{R}}=\mathcal{R}_{i}\left(u^{\mathcal{R}_{i}^{*}}, \zeta_{\mathrm{R}}-\zeta^{\mathcal{R}_{i}^{*}}\right),
$$

such that the composite curve is given by

$$
\mathcal{E}\left(u_{\mathrm{L}}, \zeta\right)= \begin{cases}\mathcal{H}_{i}\left(u_{\mathrm{L}}, \zeta\right) & \text { for } \zeta \in\left[0, \zeta^{\mathcal{R}_{i}^{*}}\right), \\ \mathcal{R}_{i}\left(u^{\mathcal{R}_{i}^{*}}, \zeta-\zeta^{\mathcal{R}_{i}^{*}}\right) & \text { for } \zeta \in\left[\zeta^{\mathcal{R}_{i}^{*}}, \zeta_{\mathrm{R}}\right] .\end{cases}
$$

In [2], the elementary curves are parametrized by the unknown solution variables. However, for a semi-analytical construction of elementary curves in phase space, a parametrization by curve lengths is more convenient. It allows the solution of a Riemann problem by concatenating elementary curves and fitting the curve length such that the projected right state coincides with the given right state.

\subsection{Characteristic fields with a finite number of linear degeneracies}

An elementary curve which passes through a finite number of MLDs, each of codimension one, can be parametrized analogously. Therefore we define $u^{\mathcal{C}_{i k}} \in \mathcal{W}_{i}\left(u_{\mathrm{L}}, \cdot\right), k=1, \ldots, K_{i}$ as the intersection points of the MLDs with the constructed elementary waves, which means that $D_{r_{i}}\left(D_{r_{i}}\left(\lambda_{i}\left(u^{\mathcal{C}_{i k}}\right)\right)\right)=0$ at these states. Assume that at the starting point $u^{\mathcal{R}_{i 1}} \in \mathcal{H}_{i}\left(u_{\mathrm{L}}, \cdot\right)$, the equation $\sigma\left(u_{\mathrm{L}}, u^{\mathcal{R}_{i 1}}\right)=\lambda_{i}\left(u^{\mathcal{R}_{i 1}}\right)$ is satisfied. Moreover, the rarefaction segments have the parametrization

$$
\begin{aligned}
& u^{\mathcal{C}_{i k}}=\mathcal{R}_{i}\left(u^{\mathcal{R}_{i k}}, \zeta^{\mathcal{C}_{i k}}-\zeta^{\mathcal{R}_{i k}}\right), \quad\left(r_{i} \cdot \nabla \lambda_{i}\right)\left(\mathcal{R}_{i}\left(u^{\mathcal{R}_{i k}}, \zeta^{\mathcal{C}_{i k}}-\zeta^{\mathcal{R}_{i k}}\right)\right)=0, \\
& \left(r_{i} \cdot \nabla \lambda_{i}\right)\left(\mathcal{R}_{i}\left(u^{\mathcal{R}_{i k}}, \zeta\right)\right) \geq 0 \quad \text { for all } \zeta \in\left[0, \zeta^{\mathcal{C}_{i k}}-\zeta^{\mathcal{R}_{i k}}\right),
\end{aligned}
$$

where the contact segments are defined as

$$
\begin{gathered}
\mathcal{C}_{i K}\left(u^{\mathcal{C}_{i K}}, \cdot\right)=\left\{u \in \mathcal{H}_{i}\left(u^{\mathcal{R}_{i k}^{\prime}}\right): u^{\mathcal{R}_{i k}^{\prime}} \in \mathcal{R}_{i 1}\left(u^{\mathcal{R}_{i 1}}, \zeta\right) \cup \cdots \cup \mathcal{R}_{i K}\left(u^{\mathcal{R}_{i K}}, \zeta\right), \zeta \in\left[0, \zeta^{\mathcal{C}_{i k}}-\zeta^{\mathcal{R}_{i k}}\right],\right. \\
\left.\exists k \in\{1, \ldots, K\}: \lambda_{i}\left(u^{\mathcal{R}_{i k}^{\prime}}\right)=\sigma\left(u^{\mathcal{R}_{i k}^{\prime}}, u\right)\right\}, \quad K \in\left\{1, \ldots, K_{i}\right\} .
\end{gathered}
$$

In (3.9) $u^{\mathcal{R}_{i k}^{\prime}} \in \mathcal{W}_{i}\left(u_{\mathrm{L}}, \cdot\right)$ is defined implicitly. The signed parametrization is assumed to be chosen such that

$$
\left.r_{i}\left(u^{\mathcal{R}_{i k}^{\prime}}\right) \cdot \partial_{\zeta} \mathcal{R}_{i k}\left(u^{\mathcal{R}_{i k}^{\prime}}, \zeta\right)\right|_{\zeta=0}>0 \quad \text { for all } k .
$$

The elementary curve is then given by

$$
\mathcal{E}_{i}\left(u_{\mathrm{L}}, \zeta\right)= \begin{cases}\mathcal{H}_{i}\left(u_{\mathrm{L}}, \zeta\right) & \text { for } \zeta \in\left[0, \zeta^{\mathcal{R}_{i k}}\right) \text { if }\left.r_{i}\left(u_{\mathrm{L}}\right) \cdot \partial_{\zeta} \mathcal{H}_{i}\left(u_{\mathrm{L}}, \zeta\right)\right|_{\zeta=0}<0 \\ \mathcal{R}_{i k}\left(u^{\mathcal{R}_{i k}}, \zeta\right) & \text { for } \zeta \in\left[0, \zeta^{\mathcal{C}_{i k}}-\zeta^{\mathcal{R}_{i k}}\right), k=1, \ldots, K_{i} \\ \mathcal{C}_{i k}\left(u^{\mathcal{C}_{i k}}, \zeta\right) & \text { for } \zeta \in\left[0, \zeta^{\mathcal{R}_{i, k+1}}-\zeta^{\mathcal{C}_{i k}}\right], k=1, \ldots, K_{i}\end{cases}
$$


If the construction both starts and finishes in the direction of decreasing eigenvalues, then

$$
\left.r_{i}\left(u_{\mathrm{L}}\right) \cdot \partial_{\zeta} \mathcal{H}_{i}\left(u_{\mathrm{L}}, \zeta\right)\right|_{\zeta=0}<0,\left.\quad r_{i}\left(u_{\mathrm{R}}\right) \cdot \partial_{\zeta} \mathcal{H}_{i}\left(u_{\mathrm{R}}, \zeta\right)\right|_{\zeta=0}<0
$$

For the parametrization we distinguish global parameters for the solution of the Riemann problem, such as the wave length, from local parameters, which describe the internal composition of an elementary wave, such as the rarefaction segment lengths and the shock strengths. All local elementary wave parameters run over two indices, namely $i=1, \ldots, N$ for the number of elementary wave and $k=1, \ldots, K_{i}$ for the number of rarefaction curve parts, where the system size $N$ is fixed and the number $K_{i}$ of rarefaction curve parts for each elementary wave is dynamic.

For convenience, the segment lengths $\xi_{i 0}^{*}:=\left\|u_{\mathrm{L} i}-u^{\mathcal{R}_{i 1}}\right\|_{2}, \xi_{i k}^{*}:=\left\|u^{\mathcal{R}_{i k}^{*}}-u^{\mathcal{R}_{i, k+1}}\right\|_{2}$ for $k=1, \ldots, K_{i}-1$ and

$$
\xi_{i k}=\int_{0}^{\xi_{i k}}\left\|\partial_{\tau} \mathcal{G}_{i}\left(u^{\mathcal{R}_{i k}}, \tau\right)\right\|_{2} d \tau, \quad k=1, \ldots, K_{i}
$$

are defined, where $\xi_{i k}^{*}$ and $\xi_{i k}$ are the local parameters for the shock strengths and the rarefaction segment lengths, respectively, and $\mathcal{G}_{i}$ is the parametrization of the rarefaction curve. To describe an elementary wave, we parametrize rarefaction curve parts $\mathcal{G}_{i}:\left(\mathbb{R}^{N}, \mathbb{R}\right) \rightarrow \mathbb{R}^{N}, i=1, \ldots, N$, as

$$
\begin{aligned}
& \mathcal{G}_{i}\left(u^{\mathcal{R}_{i k}}, 0\right)=u^{\mathcal{R}_{i k}}, \quad \partial_{\tau} \mathcal{G}_{i}\left(u^{\mathcal{R}_{i k}}, \tau\right)=r_{i}\left(\mathcal{G}_{i}\left(u^{\mathcal{R}_{i k}}, \tau\right)\right), \quad \mathcal{G}_{i}\left(u^{\mathcal{R}_{i k}}, \xi_{i k}\right)=u^{\mathcal{R}_{i k}^{*}}, \\
& r_{i}\left(\mathcal{G}_{i}\left(u^{\mathcal{R}_{i k}}, \tau\right)\right) \cdot \nabla \lambda_{i}\left(\mathcal{G}_{i}\left(u^{\mathcal{R}_{i k}}, \tau\right)\right)>0, \quad\left\|r_{i}\left(\mathcal{G}_{i}\left(u^{\mathcal{R}_{i k}}, \tau\right)\right)\right\|_{2}=1 \quad \text { for } \tau \in\left[0, \tau_{i}\right] .
\end{aligned}
$$

From the parametrization of speed-state pairs

$$
G_{i}: \tau \mapsto\left(s_{i}, g_{i}\right):=\left(\lambda_{i}\left(\mathcal{G}_{i}\left(u^{\mathcal{R}_{i k}}, \tau\right)\right), \mathcal{G}_{i}\left(u^{\mathcal{R}_{i k}}, \tau\right)\right)
$$

one can extract the map $g_{i}: \mathbb{R} \rightarrow \mathbb{R}^{N}$ of speeds onto states in phase space, which corresponds to the inverse of the first derivative of the flux function for scalar conservation laws. The composite curves are characterized as

$$
\begin{aligned}
& \mathcal{H}_{i}\left(u^{\mathcal{R}_{i k}^{*}}, 0\right)=u^{\mathcal{R}_{i k}^{*}}, \quad \mathcal{H}_{i}\left(u^{\mathcal{R}_{i k}^{*}}, \xi_{i k}^{*}\right)=u^{\mathcal{R}_{i, k+1}}, \quad k=1, \ldots, K-1, \\
& f\left(\mathcal{H}_{i}\left(u^{\mathcal{R}_{i k}^{*}}, \tau\right)\right)-f\left(u^{\mathcal{R}_{i k}^{\prime}}\right)=\sigma\left(\mathcal{H}_{i}\left(u^{\mathcal{R}_{i k}^{*}}, \tau\right), u^{\mathcal{R}_{i k}^{\prime}}\right)\left(\mathcal{H}_{i}\left(u^{\mathcal{R}_{i k}^{*}}, \tau\right)-u^{\mathcal{R}_{i k}^{\prime}}\right), \\
& \partial_{\tau} \mathcal{H}_{i}\left(u^{\mathcal{R}_{i k}^{*}}, 0\right)=-r_{i}\left(u^{\mathcal{R}_{i k}^{*}}\right), \quad \tau=\left\|\mathcal{H}_{i}\left(u^{\mathcal{R}_{i k}^{*}}, \tau\right)-\mathcal{H}_{i}\left(u^{\mathcal{R}_{i k}^{*}}, 0\right)\right\|_{2} \quad \text { for } \tau \in\left[0, \xi_{i k}^{*}\right],
\end{aligned}
$$

such that speed-state pairs can be expressed as

$$
H_{i}: \tau \mapsto\left(s_{i}, h_{i}\right)=\left(\sigma\left(\mathcal{R}_{i k}^{\prime}, \mathcal{H}_{i}\left(\mathcal{R}_{i k}^{*}, \tau\right)\right), \mathcal{H}_{i}\left(\mathcal{R}_{i k}^{*}, \tau\right)\right)
$$

The Liu entropy condition can be verified by checking whether $s_{i}\left(\xi_{i k}^{*}\right) \leq s_{i}(\tau)$ holds for all $\tau \in\left[0, \xi_{i k}^{*}\right]$. After introducing the global parameters

$$
\xi^{\mathcal{R}_{i k}}:=\sum_{j=0}^{k} \xi_{i j}^{*}+\sum_{j=1}^{k} \xi_{i j}, \quad \xi^{\mathcal{R}_{i k}^{*}}:=\xi^{\mathcal{R}_{i k}}+\xi_{i k},
$$

we can describe the states on an elementary curve depending on the curve length as follows:

$$
\mathcal{E}_{i}\left(u_{\mathrm{L} i}, \tau\right)= \begin{cases}u_{\mathrm{L} i} & \text { for } \tau=0, \\ \mathcal{H}_{i}\left(u_{\mathrm{L} i}, \tau\right) & \text { for } 0<\tau \xi_{i 0}^{*}, \\ \mathcal{G}_{i}\left(u^{\mathcal{R}_{i 1}}, \tau-\xi_{i 0}^{*}\right) & \text { for } \xi_{i 0}^{*}<\tau \leq \xi_{i 0}^{*}+\xi_{i 1}, \\ & \vdots \\ \mathcal{H}_{i}\left(u^{\mathcal{R}_{i k}^{*}}, \tau-\xi^{\mathcal{R}_{i k}^{*}}\right) & \text { for } \xi^{\mathcal{R}_{i, k-1}^{*}}<\tau \leq \xi^{\mathcal{R}_{i k}}, k=2, \ldots, K, \\ \mathcal{G}_{i}\left(u^{\mathcal{R}_{i k}}, \tau-\xi^{\mathcal{R}_{i k}}\right) & \text { for } \xi^{\mathcal{R}_{i k}}<\tau \leq \xi^{\mathcal{R}_{i k}^{*}}, k=2, \ldots, K, \\ \mathcal{H}_{i}\left(u^{\mathcal{R}_{i K}^{*}}, \tau\right) & \vdots \\ u_{\mathrm{R} i} & \text { for } \xi^{\mathcal{R}_{i K}^{*}}<\tau<\left|\ell_{i}\right|, \\ & \text { for }\left|\ell_{i}\right|=\tau .\end{cases}
$$


Here, the global parameter for the elementary wave length $\ell_{i}$ is defined as

$$
\ell_{i}=\operatorname{sgn}\left(\partial_{\tau} w_{i}\left(s_{i}(\tau=0) \cdot \nabla \lambda_{i}\left(u_{\mathrm{L} i}\right)\right)\right)\left(\sum_{j=1}^{K_{i}} \xi_{i j}+\sum_{j=0}^{K_{i}} \xi_{i j}^{*}\right), \quad i=1, \ldots, N .
$$

The states on an elementary wave (where the composite curves are dropped) depending on the propagation speed can be stated as

$$
w_{i}\left(s_{i}(\tau)\right)= \begin{cases}u_{\mathrm{L} i} & \text { for } \lambda_{i}\left(u_{\mathrm{R}, i-1}\right)<s_{i}(\tau) \leq \lambda_{i}\left(u^{\mathcal{R}_{i 1}}\right), \\ & \vdots \\ \mathcal{G}_{i}\left(u^{\mathcal{R}_{i k}}, \tau-\xi^{\mathcal{R}_{i k}}\right) & \text { for } \lambda_{i}\left(u^{\mathcal{R}_{i k}}\right)<s_{i}(\tau) \leq \lambda_{i}\left(u^{\mathcal{R}_{i k}^{*}}\right), k=2, \ldots, K, \\ & \vdots \\ u_{\mathrm{R} i} & \text { for } \lambda_{i}\left(u_{\mathrm{R} i}\right)<s_{i}(\tau) \leq \lambda_{i}\left(u_{\mathrm{L}, i+1}\right) .\end{cases}
$$

For illustration, a prototype wave is shown in Fig. 1.

(a)

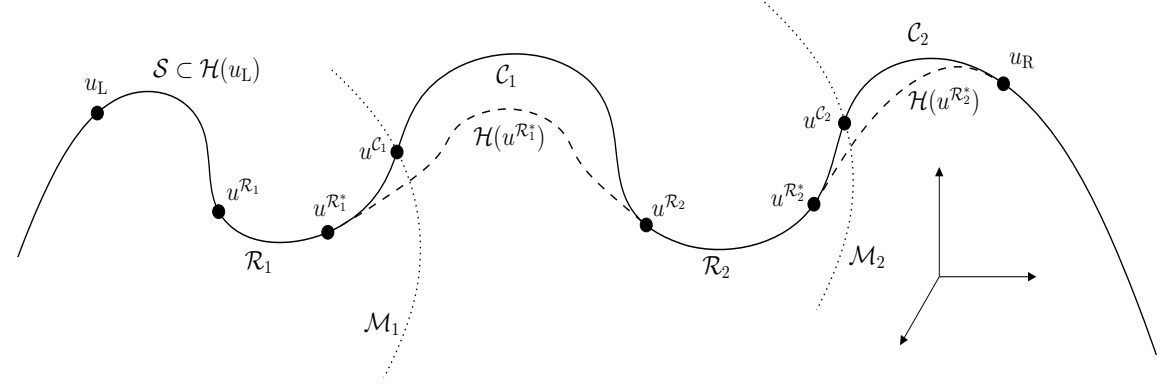

(b)

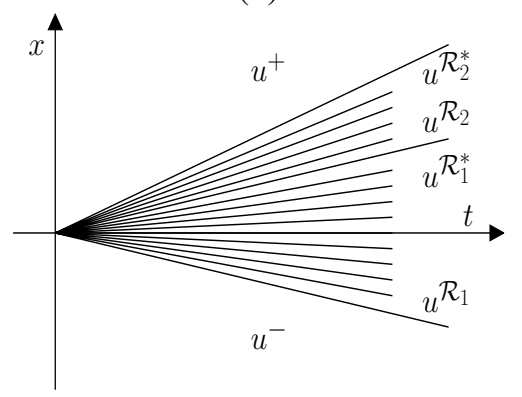

Fig. 1 Prototype wave (a) in phase space, (b) as a fan in an $x$ - $t$ diagram.

\subsection{Segments of an elementary curve}

An elementary wave is the solution of a Riemann problem with respect to a given characteristic family. An elementary curve is the set of states in phase space which can be connected to a given state by an elementary wave.

The elementary curves $\mathcal{E}_{1}, \ldots, \mathcal{E}_{N}$ of the respective characteristic families are composed of rarefaction, shock, contact and linear segments, denoted by the respective symbols $\mathcal{R}_{i k}, \mathcal{S}_{i k}, \mathcal{C}_{i k}$, and $\mathcal{L}_{i k}$ for $i=1, \ldots, N$ and $k=1, \ldots, K_{i}$. Linear segments are introduced to address systems with MLDs, which are not restricted to be of codimension one. Thus, a curve segment $\mathcal{E}_{i k} \in\left\{\mathcal{R}_{i k}, \mathcal{S}_{i k}, \mathcal{C}_{i k}, \mathcal{L}_{i k}\right\}$ can be represented as a one-dimensional manifold $\mathcal{E}_{i k}\left(u^{-}\right)=\mathcal{E}_{i k}\left(u^{-}, \cdot\right)$ passing through a point $u^{-}$, which includes the representation as a segment connecting $u^{-}$to $u^{+} \in \mathcal{E}_{i k}\left(u^{-}\right)$:

$$
\mathcal{E}_{i k}\left(u^{-}, u^{+}\right)=\mathcal{E}_{i k}\left(u^{-}, \xi^{+}\right)=\left\{u \in \mathcal{E}_{i k}\left(u^{-}\right): \xi^{-} \leq \xi \leq \xi^{+}\right\} .
$$

An elementary curve $\mathcal{E}_{i}$ is a concatenation of $K_{i}$ curve segments, $\mathcal{E}_{i}=\mathcal{E}_{i 1} \circ \cdots \circ \mathcal{E}_{i K_{i}}$. We use the abbreviation $\mathcal{E}_{i}:=$ $\mathcal{E}_{i}\left(\left(u^{-}\right)^{(i)},\left(u^{+}\right)^{(i)}\right), i=1, \ldots, N$ and assume $\left(u^{+}\right)^{(i)}=\left(u^{-}\right)^{(i+1)}, i=1, \ldots, N-1$. We refer to a neighboring state by $u_{ \pm}^{\mathcal{E}_{i}}:=u^{\mathcal{E}_{i}} \pm \varepsilon r\left(u^{\mathcal{E}_{i}}\right)$ for small $\varepsilon>0$.

In this subsequent subsections, all curve segment types, $\mathcal{R}_{i k}, \mathcal{S}_{i k}, \mathcal{C}_{i k}$, and $\mathcal{L}_{i k}$, are characterized along with all possible transitions between them. We denote by $u^{-}=\mathcal{E}_{i}\left(u^{-}, 0\right)$ the left state of an elementary $i$-curve $\mathcal{E}_{i}\left(u^{-}, \cdot\right)$ and by $u^{\diamond}=$ $\mathcal{E}_{i}\left(u^{-}, \zeta^{\diamond}\right)$ any state on the curve. The curve $\mathcal{E}_{i}\left(u^{-}\right)$and the local eigenvalues $\lambda_{i}$ along it are always considered in the direction of increasing $\zeta$. For each segment type, an entrance condition and a type characterization are given. The wave construction starts at a state $u_{\mathrm{L} i}$. The first segment is either a shock, a rarefaction or a linear segment, depending on whether $\lambda_{i}$ decreases, increases, or remains constant in the construction direction. The states at which a rarefaction, shock, contact or linear wave curve segment starts, are denoted by $u^{\mathcal{R}_{i k}}, u^{\mathcal{S}_{i k}}, u^{\mathcal{C}_{i k}}$, and $u^{\mathcal{L}_{i k}}$, respectively. The construction direction is always towards the increasing absolute value of the curve length $|\zeta|$, with $\zeta=0$ at the anchor state. The sign of $\zeta$ is associated with the branch of construction. 
(a)

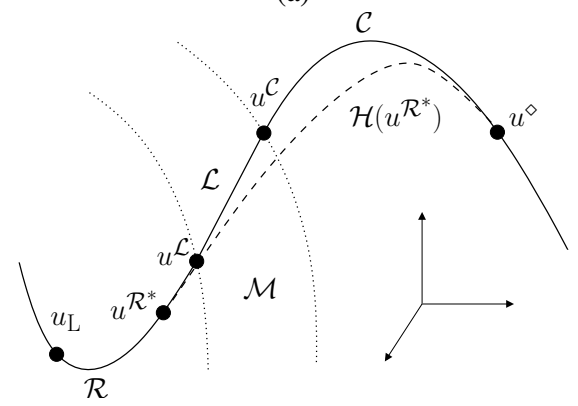

(b)

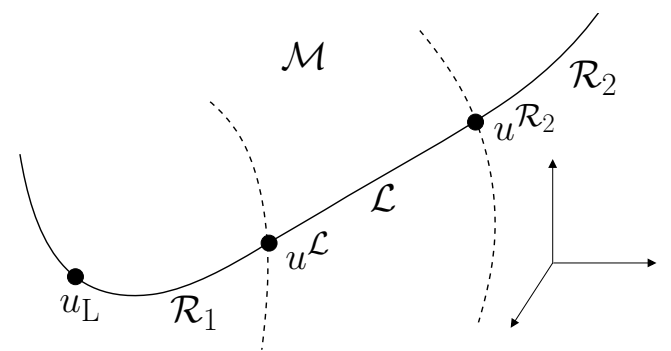

Fig. 2 Phase space plots of linear segments followed by (a) a contact and (b) a rarefaction segment.

\subsubsection{Rarefaction segments}

A rarefaction segment $\mathcal{R}_{i k}\left(u^{\mathcal{R}_{i k}}, u^{\mathcal{L}_{i k}}\right)$ is characterized by increasing eigenvalues, i.e.,

$$
r_{i}(u) \cdot \nabla \lambda_{i}(u)>0 \quad \text { for all } u \in \mathcal{R}_{i k}\left(u^{\mathcal{R}_{i k}}, u^{\mathcal{L}_{i k}}\right),
$$

and the condition that either $u^{\mathcal{R}_{i k}}=u_{\mathrm{L} i}$ (when the construction is started) or $u^{\mathcal{R}_{i k}}$ is connected backward to a state $u^{\mathcal{R}_{i, k-1}^{\prime}}$ as defined in (3.9) on a previously constructed rarefaction segment with

$$
\lambda_{i}\left(u^{\mathcal{R}_{i, k-1}^{\prime}}\right)=\sigma\left(u^{\mathcal{R}_{i, k-1}^{\prime}}, u^{\mathcal{R}_{i k}}\right)=\lambda_{i}\left(u^{\mathcal{R}_{i k}}\right),
$$

when a contact segment precedes, or $\sigma\left(u_{\mathrm{L} i}, u^{\mathcal{R}_{i k}}\right)=\lambda_{i}\left(u^{\mathcal{R}_{i k}}\right)$, when a shock segment precedes. The backward state $u^{\mathcal{R}_{i, k-1}^{\prime}}$ depends on the preceding curve segment type $\mathcal{R}_{i k}, \mathcal{L}_{i k}$ or $\mathcal{C}_{i k}$. The entrance condition for a rarefaction segment $\mathcal{R}_{i k}$ at a point $u^{\mathcal{R}_{i k}}$ is

$$
\sigma\left(u^{\mathcal{R}_{i, k-1}^{\prime}}, u^{\mathcal{R}_{i k}}\right)=\lambda_{i}\left(u^{\mathcal{R}_{i k}}\right), \quad r\left(u_{+}^{\mathcal{R}_{i k}}\right) \cdot \nabla \lambda_{i}\left(u_{+}^{\mathcal{R}_{i k}}\right)>0,
$$

which guarantees the entropy condition. In the entrance state $u^{\mathcal{R}_{i k}}$ the shock speed on the preceding Hugoniot locus starts to exceed $\lambda_{i}(u)$. The subindex " + " is only needed if a linear segment precedes, along which $r_{i}(u) \cdot \nabla \lambda_{i}(u)=0$ is valid. While $\lambda_{i}(u(\zeta))$ increases in the construction direction, a rarefaction wave can be pursued. The characteristic speed rate remains then positive on the rarefaction segment. When crossing a MLD, a rarefaction segment is left and the segment type changes to linear. If $\lambda_{i}$ starts to decrease, the linear segment $\mathcal{L}_{i k}\left(u^{\mathcal{L}_{i k}}, u^{\mathcal{M}_{i k}}\right)$ is followed by a contact discontinuity segment, but if $\lambda_{i}$ starts to increase again, then $\mathcal{L}_{i k}\left(u^{\mathcal{L}_{i k}}, u^{\mathcal{M}_{i k}}\right)$ is followed by another rarefaction segment.

\subsubsection{Shock segments}

A shock segment is started in the left state $u_{\mathrm{Li}}$ if $\lambda_{i}$ decreases in the construction direction. A point $u^{\diamond} \in \mathcal{S}_{i k}\left(u^{\mathcal{S}_{i k}}\right)$ on a shock segment starting at $u^{\mathcal{S}_{i k}}=u_{\mathrm{L} i}$ is characterized by the admissibility condition

$$
\sigma\left(u_{\mathrm{L} i}, \zeta^{\diamond}\right) \leq \sigma\left(u_{\mathrm{L}}, \zeta\right) \quad \text { for all } \zeta \in\left[\zeta_{\mathrm{L} i}, \zeta^{\diamond}\right]
$$

A shock segment is left if a state $u^{\mathcal{R}_{i k}}$ is reached at which the equality $\sigma\left(u_{\mathrm{L} i}, u^{\mathcal{R}_{i k}}\right)=\lambda_{i}\left(u^{\mathcal{R}_{i k}}\right)$ holds. The rate of the shock speed enforces the entrance of a rarefaction segment. For $u \diamond \in \mathcal{H}_{i}\left(u_{\mathrm{L} i}, u^{\mathcal{R}_{i k}}\right)$ the shock admissibility criterion $\sigma\left(u_{\mathrm{L} i}, u^{\diamond}\right) \leq \sigma\left(u_{\mathrm{L} i}, u\right)$ for all $u \in\left[u_{\mathrm{L} i}, u^{\diamond}\right]$ is satisfied, but it is not satisfied on the Hugoniot locus beyond $u^{\mathcal{R}_{i k}}$.

A shock segment $\mathcal{S}_{i k}$ can also be entered during the construction of a contact segment when the backtracking anchor on rarefactions backs up to the original left state, $u^{\mathcal{R}_{i k}^{\prime}}=u_{\mathrm{L} i}$.

\subsubsection{Segments of linear degeneracy}

If the MLD is not restricted to be of codimension one, then segments of linear degeneracy can occur. The entrance condition for a linearly degenerate segment $\mathcal{L}_{i k}$ is that $\nabla \lambda_{i}(u) \cdot r_{i}(u)$ vanishes after being positive, i.e. $r_{i}\left(u^{\mathcal{L}_{i k}}\right) \cdot \nabla \lambda_{i}\left(u^{\mathcal{L}_{i k}}\right)>0$ but $r_{i}\left(u^{\mathcal{L}_{i k}}\right) \cdot \nabla \lambda_{i}\left(u^{\mathcal{L}_{i k}}\right)=0$.

The only situation in which a segment of linear degeneracy $\mathcal{L}_{i k}$, which is embedded in a MLD $\mathcal{M}_{i}$ and starts at a state $u^{\mathcal{L}_{i k}}$, affects the construction of the elementary curve occurs when $u^{\mathcal{L}_{i k}}$ also is the exit state of a rarefaction segment. (In the other cases, namely, when a shock or a contact discontinuity curve crosses a MLD, the wave does not change type 
since the Liu entropy condition, which has to hold along wave curves of that type, does not depend on the speed rate $r_{i}(u) \cdot \nabla \lambda_{i}(u)$.) In this case, the continuation of the construction of the composed wave depends on the speed rate near the state $u^{\mathcal{M}_{i k}}$ marking the exit of the linear segment. This state is the entrance either to a new rarefaction wave or to a new contact segment (Fig. 2 (a) $u^{\mathcal{M}_{i k}}=u^{\mathcal{R}_{i k}}$, (b) $u^{\mathcal{M}_{i k}}=u^{\mathcal{C}_{i k}}$ ) according to the following criterion:

$$
u^{\mathcal{M}_{i k}}= \begin{cases}u^{\mathcal{R}_{i k}} & \text { if } r_{i}\left(u_{+}^{\mathcal{M}_{i k}}\right) \cdot \nabla \lambda_{i}\left(u_{+}^{\mathcal{M}_{i k}}\right)>0 \\ u^{\mathcal{C}_{i k}} & \text { if } r_{i}\left(u_{+}^{\mathcal{M}_{i k}}\right) \cdot \nabla \lambda_{i}\left(u_{+}^{\mathcal{M}_{i k}}\right)<0 .\end{cases}
$$

\subsubsection{Contact segments}

A contact segment starting in $u^{\mathcal{C}_{i k}}$ is entered when $r_{i}(u) \cdot \nabla \lambda_{i}(u)$ becomes negative, i.e.

$$
r\left(u^{\mathcal{C}_{i k}}\right) \cdot \nabla \lambda_{i}\left(u^{\mathcal{C}_{i k}}\right) \geq 0, \quad r\left(u_{+}^{\mathcal{C}_{i k}}\right) \cdot \nabla \lambda_{i}\left(u_{+}^{\mathcal{C}_{i k}}\right)<0 .
$$

A state $u^{\diamond}$ on a contact segment has to be constructed such that the admissibility criterion

$$
\sigma\left(u^{\mathcal{R}_{i k}^{\prime}}, u^{\diamond}\right) \leq \sigma\left(u^{\mathcal{R}_{i k}^{\prime}}, u\right) \quad \text { for all } u \in \mathcal{H}\left(u^{\mathcal{R}_{i k}^{\prime}}, u^{\diamond}\right)
$$

is satisfied, i.e., from some $u^{\mathcal{R}_{i k}^{\prime}}$ on a past rarefaction, a Hugoniot locus is constructed until $\lambda_{i}\left(u^{\mathcal{R}_{i k}^{\prime}}\right)=\sigma\left(u^{\mathcal{R}_{i k}^{\prime}}, u^{\diamond}\right)<$ $\lambda_{i}\left(u^{\diamond}\right)$. The right inequality indicates the exit condition $\sigma\left(u^{\mathcal{R}_{i k}^{\prime}}, u^{\diamond}\right)=\lambda_{i}\left(u^{\diamond}\right)$.

The crucial part in constructing waves for systems appears when a region in phase space with increasing characteristic speeds is adjacent to a region with decreasing speeds. The issue is to continue a rarefaction segment $\mathcal{R}_{i k}\left(u^{\mathcal{R}_{i k}}, u^{\mathcal{L}_{i k}}\right)$, where $r_{i}(u) \cdot \nabla \lambda_{i}(u)>0$ for $u=u(\zeta)$ on $\zeta \in\left(\zeta^{\mathcal{R}_{i k}}, \zeta^{\mathcal{L}_{i k}}\right)$, followed by a linear segment $\mathcal{L}_{i k}\left(u^{\mathcal{L}_{i k}}, u^{\mathcal{C}_{i k}}\right)$, beyond the point $u_{i k}^{\mathcal{C}}$ if

$$
r_{i}\left(u^{\mathcal{C}_{i k}}\right) \cdot \nabla \lambda_{i}\left(u^{\mathcal{C}_{i k}}\right)=0, \quad r_{i}\left(u_{+}^{\mathcal{C}_{i k}}\right) \cdot \nabla \lambda_{i}\left(u_{+}^{\mathcal{C}_{i k}}\right)<0
$$

The construction of the contact segment ("mixed wave" in [36]) is as follows: Take a point $u^{\mathcal{R}_{i k}^{\prime}} \in \mathcal{R}_{i k}\left(u_{\mathrm{L} i}, u^{\mathcal{L}_{i k}}\right) \cap$ $B\left(u^{\mathcal{L}_{i k}}, \varepsilon\right)$ in a sufficiently small neighborhood $B\left(u^{\mathcal{L}_{i k}}, \varepsilon\right)$ of $u^{\mathcal{L}_{i k}}$. Construct the Hugoniot locus $\mathcal{H}_{i}\left(u^{\mathcal{R}_{i k}^{\prime}}\right)$ in the original wave direction $r_{i}\left(u^{\mathcal{R}_{i k}^{\prime}}\right)$ until the first point $u^{\diamond} \in \mathcal{H}_{i}\left(u^{\mathcal{R}_{i k}^{\prime}}\right)$ is met at which $\sigma\left(u^{\mathcal{R}_{i k}^{\prime}}, u^{\diamond}\right)=\lambda_{i}\left(u^{\mathcal{R}_{i k}^{\prime}}\right)$. That state $u^{\diamond}$ belongs to $\mathcal{C}_{i k}\left(u^{\mathcal{C}_{i k}}, \cdot\right)$. As noted in [36], such a state $u^{\diamond}$ exists for small $\varepsilon$ since $\sigma_{i}\left(u^{\mathcal{R}_{i k}^{\prime}}, \cdot\right)$ begins to decrease (after first increasing) when passing the MLD through $u^{\mathcal{C}_{i k}}$. The construction of a contact segment and its analogue for a scalar equation are depicted in Fig. 3.

(a)

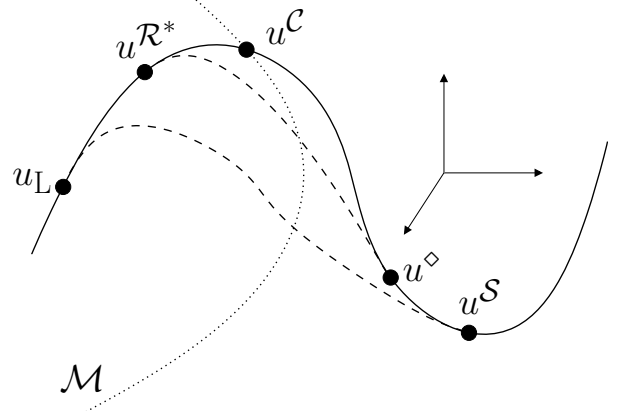

(b)

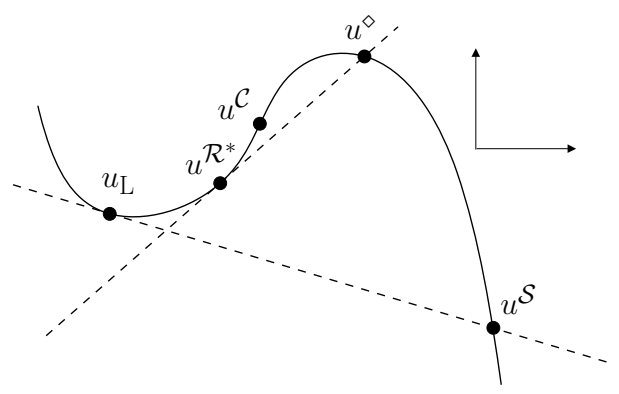

Fig. 3 Contact segment followed by shock segment (a) in phase space and (b) its analogue for a scalar equation.

We now proceed to the problem of leaving a contact segment. When selecting a state $u^{\mathcal{R}_{i, k-1}^{\prime}}$ on the union of the already obtained rarefaction segments, on which the eigenvalues decrease towards the backward direction, two cases are possible. In the first, a shock segment is entered, where $u^{\mathcal{R}_{i, k-1}^{\prime}}=u_{\mathrm{L} i}$ and $\lambda_{i}\left(u_{\mathrm{L} i}\right)=\sigma\left(u_{\mathrm{L} i}, u^{\diamond}\right)$. In the second, another MLD $\mathcal{N}$ is crossed. After leaving $\mathcal{N}$, the eigenvalues increase again. Here, a state $u^{\diamond}$ is reached where $\lambda_{i}\left(u^{\mathcal{R}_{i k}^{\prime}}\right)=\sigma\left(u^{\mathcal{R}_{i k}^{\prime}}, u^{\diamond}\right)=$ $\lambda_{i}\left(u^{\diamond}\right)$, and thus the elementary curve enters a rarefaction segment. 
Table 1 Entrance, state, and exit conditions for segments of composed waves. Arrows indicate possible subsequent segment types.

\begin{tabular}{|c|c|c|c|}
\hline Type & Entrance condition & State condition & Exit condition \\
\hline $\mathcal{R}_{i k}$ & $\lambda_{i}\left(u^{\mathcal{R}_{i, k-1}^{\prime}}\right)=\sigma\left(u^{\mathcal{R}_{i, k-1}^{\prime}}, u^{\mathcal{R}_{i k}}\right)=\lambda_{i}\left(\mathcal{R}_{i k}\right)$ & $r_{i}\left(u^{\diamond}\right) \cdot \nabla \lambda_{i}\left(u^{\diamond}\right)>0$ & $r_{i}\left(u^{\diamond}\right) \cdot \nabla \lambda_{i}\left(u^{\diamond}\right)=0 \quad\left(\rightarrow \mathcal{L}_{i k}, \mathcal{R}_{i k}\right)$ \\
\hline $\mathcal{S}_{i k}$ & $u^{\mathcal{R}_{i k}^{\prime}}=u_{\mathrm{L}}$ & $\begin{array}{l}\forall u \in \mathcal{H}\left(u_{\mathrm{L}}, u^{\diamond}\right) \\
\sigma\left(u_{\mathrm{L}}, u^{\diamond}\right) \leq \sigma\left(u_{\mathrm{L}}, u\right)\end{array}$ & $\sigma\left(u_{\mathrm{L}}, u^{\diamond}\right)=\lambda_{i}\left(u^{\diamond}\right) \quad\left(\rightarrow \mathcal{R}_{i k}\right)$ \\
\hline $\mathcal{C}_{i k}$ & $\begin{array}{l}r_{i}\left(u_{-}^{\mathcal{C}_{i k}}\right) \cdot \nabla \lambda_{i}\left(u_{-}^{\mathcal{C}_{i k}}\right) \geq 0 \\
r_{i}\left(u_{+}^{\mathcal{C}_{i k}}\right) \cdot \nabla \lambda_{i}\left(u_{+}^{\mathcal{C}_{i k}}\right)<0\end{array}$ & $\begin{array}{l}\lambda_{i}\left(u^{\mathcal{R}_{i k}^{\prime}}\right) \\
=\sigma\left(u^{\mathcal{R}_{i k}^{\prime}}, u^{\diamond}\right)>\lambda_{i}\left(u^{\diamond}\right)\end{array}$ & $\begin{array}{l}\sigma\left(u_{\mathcal{R}_{i k}^{\prime}}^{\prime} u^{\diamond}\right)=\lambda_{i}\left(u^{\diamond}\right) \quad\left(\rightarrow \mathcal{R}_{i k}\right) \\
u^{\mathcal{R}_{i k}^{\prime}}=u_{\mathrm{L}} \quad\left(\rightarrow \mathcal{S}_{i k}\right)\end{array}$ \\
\hline $\mathcal{L}_{i k}$ & $\begin{array}{l}r\left(u_{i}^{\mathcal{L}_{i k}}\right) \cdot \nabla \lambda_{i}\left(u_{i}^{\mathcal{L}_{i k}}\right)>0 \\
r_{i}\left(u^{\mathcal{L}_{i k}}\right) \cdot \nabla \lambda_{i}\left(u^{\mathcal{L}_{i k}}\right)=0\end{array}$ & $r_{i}\left(u^{\diamond}\right) \cdot \nabla \lambda_{i}\left(u^{\diamond}\right)=0$ & $\left(r_{i} \cdot \nabla \lambda_{i}\right)\left(u_{+}\right)= \begin{cases}>0 & \left(\rightarrow \mathcal{R}_{i k}\right) \\
<0 & \left(\rightarrow \mathcal{C}_{i k}\right)\end{cases}$ \\
\hline
\end{tabular}

\subsubsection{Change of wave type table, curve segment paths, entrance, and exit modes}

Entrance conditions, state characterizations, and exit conditions are summarized for all curve segment types in Table 1 . The entropy condition is satisfied on $\mathcal{S}, \mathcal{R}, \mathcal{L}$ due to the wave type and on $\mathcal{C}$ due to construction. In Table 1 all possible curve segment paths can be identified. A shock segment can only follow a contact segment and only be followed by a rarefaction segment. Rarefaction segments can alternate with linear segments. A contact segment always follows a linear segment and is either followed by a rarefaction or a shock segment.

\section{Semi-analytical solution of Riemann problems}

\subsection{Riemann problem solver}

The procedure for locally solving the Riemann problem for strictly hyperbolic systems mainly consists in solving a nonlinear system of equations, which determines the optimal lengths of the assembled elementary waves. A solution of the Riemann problem with left state $u_{\mathrm{L}}$ and right state $u_{\mathrm{R}}$ is obtained by solving

$$
u_{\mathrm{R}}-\hat{u}_{\mathrm{R}}(\ell)=0, \quad \ell:=\left(\ell_{1}, \ldots, \ell_{N}\right),
$$

where $\ell$ denotes the elementary wave lengths and an approximation of $u_{\mathrm{R}}$ by elementary waves is given by

$$
\hat{u}_{\mathrm{R}}(\ell)=\mathcal{W}_{N}\left(\mathcal{W}_{N-1}\left(\ldots \mathcal{W}_{2}\left(\mathcal{W}_{1}\left(u_{\mathrm{L}}, \ell_{1}\right), \ell_{2}\right) \ldots, \ell_{N-1}\right), \ell_{N}\right)
$$

The single parameters $\ell_{1}, \ldots, \ell_{N}$ capture the lengths of elementary waves by connecting the intermediate states as $u_{\mathrm{R} i}=\mathcal{W}_{i}\left(u_{\mathrm{L} i}, \ell_{i}\right), i=1, \ldots, N$, where $u_{\mathrm{L} i}$ and $u_{\mathrm{R} i}$ are the left and right states of the $i$-th elementary wave, respectively, which are connected as $u_{\mathrm{L}, i+1}=u_{\mathrm{R} i}$ for $i=1, \ldots, N-1$ and related to the global left and right states as $u_{\mathrm{L} 1}=u_{\mathrm{L}}$ and $u_{\mathrm{R} N}=u_{\mathrm{R}}$, respectively. Each elementary wave in turn consists of a sequence of jumps and rarefaction waves. To measure the lengths of these elementary wave parts, the rarefaction segments contribute their curve length, whereas shock or contact segments contribute the shock strength, i.e. the distance between the states on each side of the shock.

For non-strictly hyperbolic systems, the proposed Riemann solver is possibly not applicable due to non-local properties that emerge in the phase space analysis. For example, during the calculation of the displacement path in a model of three-component, three-phase flow through porous media, detached branches of the Hugoniot locus appear [18, Fig. 1]. This property is strongly connected to the intersection of integral curves of one family in the umbilic point, at which the eigenvalues of the Jacobian of the flux function coincide [17, Fig. 6]. For the solution of Riemann problems in non-strictly hyperbolic systems, the classification done for quadratic flux functions [23, 24] needs to be costumized. The proposed algorithm can also not be applied even for strictly hyperbolic system in the absence of a classical Riemann solution [29,35]. In fact, for polydisperse models, in the phase space, a detached Hugoniot locus of the origin (corresponding to zeroconcentration) can be identified to be the phase space boundary corresponding to maximal concentration; moreover it could not be formally shown that the wave speeds have increasing order (i.e., the wave speed of the second family exceeds that of the first). However, the topology of integral curves and any Hugoniot locus in the phase space appears to be independent of the size parameter (Fig. 7 (b)), and permits a solution for arbitrary initial data (Fig. 12). Unfortunately, due to the lack of closed-form expressions for the trajectories in phase space, it could not be shown rigorously (but is conjectured) that there is a Riemann solution for arbitrary left and right size within the model framework presented (the MLB model for 
equal-density particles). The proposed semi-analytical strategy is expected to be successful as soon as there is a classical solution, independently from the availability of explicit expressions of the elementary waves.

Finally, we mention that our approach is restricted to finding the Riemann solutions necessary for each case, since we do not provide a general Riemann solver in the sense of a closed-form expression of the solution for all left and right states. Our interest in the front tracking approach for the present problem arose from the need to devise an alternative solution to finite differencing, but the lack of closed-form Riemann solutions clearly limits the applicability of front tracking in this case, and finite difference methods will, in general, be more versatile.

\subsection{Semi-analytical construction of elementary waves}

In light of the discussion of Sect. 3, we now demonstrate how an elementary wave with left state $u_{\mathrm{L} i}$ and signed length $\ell_{i}$ can be constructed. The signed length indicates the orientation of an elementary wave. For a genuinely non-linear characteristic family, $\ell_{i}>0$ indicates a construction in the direction of increasing eigenvalues, while $\ell_{i}<0$ indicates a construction in the opposite direction, see (3.10). For a non-convex family, the ambiguity of direction at extremal eigenvalues along a characteristic family is avoided by enforcing the construction direction $\operatorname{dir}_{i}$ if necessary, which guarantees the orientation of the signed curve length $\operatorname{sgn}\left(\ell_{i}\right)$.

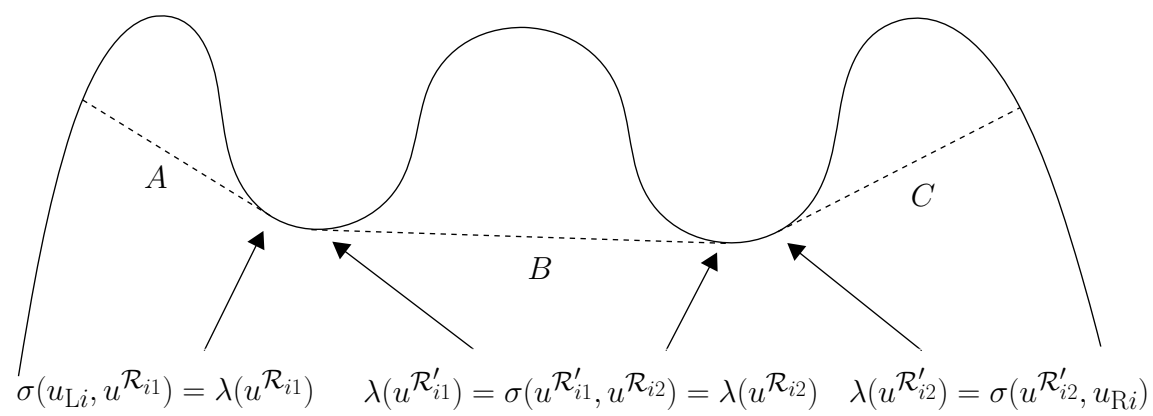

Fig. 4 Tangent construction during Algorithm 4.1.

An elementary wave is the convex hull with respect to the propagation speeds of the underlying elementary curve such that the speed increases on the convex hull. In its most general form, this convex hull has the structure given in Fig. 4. To begin with, in addition to the state $u_{\mathrm{L} i}$, the convex hull consists of that state $u^{\mathcal{R}_{i 1}} \in \mathcal{H}_{i}\left(u_{\mathrm{L} i}\right)$ that satisfies $\sigma\left(u_{\mathrm{L} i}, u^{\mathcal{R}_{i 1}}\right)=\lambda\left(u^{\mathcal{R}_{i 1}}\right)$. Then we append a sequence of rarefaction waves, which are separated by double-sided contact discontinuities, each satisfying

$$
\lambda\left(u^{\mathcal{R}_{i k}^{\prime}}\right)=\sigma\left(u^{\mathcal{R}_{i k}^{\prime}}, u^{\mathcal{R}_{i, k+1}}\right)=\lambda\left(u^{\mathcal{R}_{i, k+1}}\right), \quad k=1,2, \ldots, K-1 .
$$

Finally, a rarefaction curve followed by a one-sided contact discontinuity satisfying $\lambda\left(u^{\mathcal{R}_{i K}^{\prime}}\right)=\sigma\left(u^{\mathcal{R}_{i K}^{\prime}}, u_{\mathrm{R} i}\right)$ is appended. This construction is simplified if $u_{\mathrm{L} i}=u^{\mathcal{R}_{i 1}}$ or $u_{\mathrm{R} i}=u^{\mathcal{R}_{i K}^{\prime}}$.

The algorithmic procedure is to find first a shock transition between $u_{\mathrm{L} i}$ and an approximate right state, which may violate the entropy criterion, and then to stretch step by step the convex hull. This is done by solving a series of nonlinear equations for the shocks or contact discontinuities. For each rarefaction wave segment, a system of ordinary differential equation is solved to obtain the rarefaction curve. Algorithm 4.1 computes an elementary wave of characteristic family $i$. The wave leaves $u_{\mathrm{L} i}$ in the construction direction $\operatorname{dir}_{i}$ and has the prescribed wave length $\left|\ell_{i}\right|$. During the construction procedure, $\hat{\zeta}$ denotes the length of the already constructed wave. The rarefaction curves are computed numerically by solving the ordinary differential equation $u(\xi)^{\prime}=r(u(\xi)), u\left(\xi^{\prime}\right)=u^{\prime}$ with step-size $\delta$ as long as $\hat{\zeta} \leq\left|\ell_{i}\right|$, or until some $u^{\mathcal{C}_{i k}}$ is crossed where $(r \cdot \nabla \lambda)\left(u^{\mathcal{C}_{i k}}\right)=0$.

Algorithm 4.1 (Elementary wave $\mathcal{W}_{i}\left(u, \ell_{i}\right)$ )

if $\left(\operatorname{dir}_{i} \cdot \nabla \lambda_{i}\right)\left(u_{\mathrm{L} i}\right)<0$ then

1. Find $u^{\prime}$ of shock transition with strength $\left|\ell_{i}\right|$ on the active branch of the Hugoniot locus by solving $f\left(u^{\prime}\right)-f\left(u_{\mathrm{L} i}\right)=s\left(u^{*}-u_{\mathrm{L} i}\right),\left\|u^{*}-u_{\mathrm{L} i}\right\|_{2}=\left|\ell_{i}\right|$.

2. if entropy_check $\left(u_{\mathrm{L} i}, u^{*}\right)=$ false then

Calculate the tangential state $u^{\mathcal{R}_{i 1}} \in \mathcal{H}\left(u_{\mathrm{L} i}, \cdot\right)$, where $\sigma\left(u_{\mathrm{L} i}, u^{\mathcal{R}_{i 1}}\right)=\lambda\left(u^{\mathcal{R}_{i 1}}\right)$, solving else $f\left(u^{\mathcal{R}_{i 1}}\right)-f\left(u_{\mathrm{L} i}\right)=s\left(u^{\mathcal{R}_{i 1}}-u_{\mathrm{L} i}\right), s=\lambda\left(u^{\mathcal{R}_{i 1}}\right)$. 
stop
endif

3. Calculate rarefaction from $u^{\mathcal{R}_{i 1}}$.

if $\hat{\zeta}=\left|\ell_{i}\right|$ then stop endif

else

1. Calculate rarefaction from $u_{\mathrm{L} i}$.

2. if $\hat{\zeta}=\left|\ell_{i}\right|$ then stop endif

3. $u^{\mathcal{R}_{i 1}} \leftarrow u_{\mathrm{L} i}$

endif

$k \leftarrow 1$

while entropy_check $\left(u^{\mathcal{C}_{i k}}, u^{*}\right)=$ false $\mathbf{d o}$

1. Find $u^{\mathcal{R}_{i k}^{\prime}}$ on the rarefaction which starts in $u^{\mathcal{R}_{i k}}$ and is parametrized by $\zeta$ and then find $u^{\mathcal{R}_{i, k+1}} \in \mathcal{H}\left(u^{\mathcal{R}_{i k}^{\prime}}\right)$ on the Hugoniot locus starting in $u^{\mathcal{R}_{i k}^{\prime}}$, such that $\lambda\left(u^{\mathcal{R}_{i k}^{\prime}}\right)=\sigma\left(u^{\mathcal{R}_{i k}^{\prime}}, u^{\mathcal{R}_{i, k+1}}\right)=\lambda\left(u^{\mathcal{R}_{i, k+1}}\right)$ by solving

$$
\begin{aligned}
& f\left(u^{\mathcal{R}_{i k}^{\prime}(\zeta)}\right)-f\left(u^{\mathcal{R}_{i, k+1}}\right)=s\left(u^{\mathcal{R}_{i k}^{\prime}}-u^{\mathcal{R}_{i, k+1}}\right), \\
& s=\lambda\left(u^{\mathcal{R}_{i k}^{\prime}}(\zeta)\right), \quad s=\lambda\left(u^{\mathcal{R}_{i, k+1}}\right) .
\end{aligned}
$$

2. $k \leftarrow k+1$

3. Compute rarefaction curve from $u^{\mathcal{R}_{i k}}$.

4. if $\hat{\zeta}=\left|\ell_{i}\right|$ then stop endif

5. Find $u^{*}$ of shock transition with strength $\left|\ell_{i}\right|-\hat{\zeta}$ on the active branch of the Hugoniot locus by solving $f\left(u^{*}\right)-$ $f\left(u^{\mathcal{C}_{i k}}\right)=s\left(u^{*}-u^{\mathcal{C}_{i k}}\right),\left\|u^{*}-u^{\mathcal{C}_{i k}}\right\|_{2}=\left|\ell_{i}\right|-\hat{\zeta}$.

endwhile

A left tangent to the state $\hat{u}_{\mathrm{R} i}(\xi)$, where $\lambda\left(u^{\mathcal{R}_{i, k-1}^{\prime}}\right)=\sigma\left(u^{\mathcal{R}_{i, k-1}^{\prime}}, \hat{u}_{\mathrm{R} i}\right)$ and $u^{\mathcal{R}_{i, k-1}^{\prime}}$ on the rarefaction through $u^{\mathcal{R}_{i, k-1}}$ is found by solving

$$
f\left(u^{\mathcal{R}_{i, k-1}^{\prime}}\right)-f\left(\hat{u}_{\mathrm{R} i}\right)=s\left(u^{\mathcal{R}_{i, k-1}^{\prime}}-\hat{u}_{\mathrm{R} i}\right), \quad s=\lambda\left(u^{\mathcal{R}_{i, k-1}^{\prime}}(\zeta)\right), \quad \hat{\zeta}=\left|\ell_{i}\right| .
$$

The convex hull between the left state $u_{\mathrm{L} i}$ and the preliminary right state $\tilde{u}_{\mathrm{R} i} \in \mathcal{H}\left(u_{\mathrm{L} i}, \zeta\right)$ with $\left\|u_{\mathrm{L} i}-\tilde{u}_{\mathrm{R} i}\right\|_{2}=\left|\ell_{i}\right|$ is obtained by iteratively checking the entropy condition. To this end, the Hugoniot locus is computed, the shock speeds are compared and it is indicated whether the entropy condition is satisfied. The farthest state on $\mathcal{H}\left(u_{\mathrm{L} i}, \zeta\right)$ (with maximum distance $\hat{\zeta}$ which satisfies the entropy condition) can be used for further constructing the convex hull.

\section{Algorithm 4.2 (Entropy check)}

Calculate the tangent parameter $\zeta$ as

$$
\operatorname{argmin}_{\zeta}\left\{\sigma\left(u_{\mathrm{L} i}, u(\zeta)\right), u(\zeta) \in \mathcal{H}\left(u_{\mathrm{L} i}, \cdot\right): \sigma\left(u_{\mathrm{L} i}, u(\zeta)\right) \leq \sigma\left(u_{\mathrm{L} i}, u_{\mathrm{R} i}\right),\left\|u(\zeta)-u_{\mathrm{L} i}\right\|_{2} \leq\left\|u_{\mathrm{L} i}-u_{\mathrm{R} i}\right\|_{2}\right\} .
$$

if $\zeta=\left|\ell_{i}\right|$ then

return entropy_check = true (There is no entropy violation such that the already calculated shock is valid.)

else

return entropy_check $=$ false (The entropy condition is violated and an iterative procedure to find the convex hull is started.)

endif

\section{Modes of sedimentation for bidisperse suspensions}

\subsection{Example 1: Sedimentation of a monodisperse suspension}

The initial value problem (2.1), (2.8) models the settling of an initially homogeneous suspension of concentration $\Phi_{0}$ in a column of height $L$. In the scalar case ( $N=1$ species), we replace $\Phi, \Phi_{0}$, and $\Phi_{\infty}$ by $\phi, \phi_{0}$, and $\phi_{\infty}$, respectively. For $N=1$, the (scalar) flux function $f$, which describes the material properties and is assumed to be Lipschitz continuous with $f(\phi)>0$ for $\phi \in\left(0, \phi_{\infty}\right)$ and $f(\phi)=0$ for $\phi \leq 0$ or $\phi \geq \phi_{\infty}$ [31]. The modes of sedimentation classify the solution 
(a) flux plot

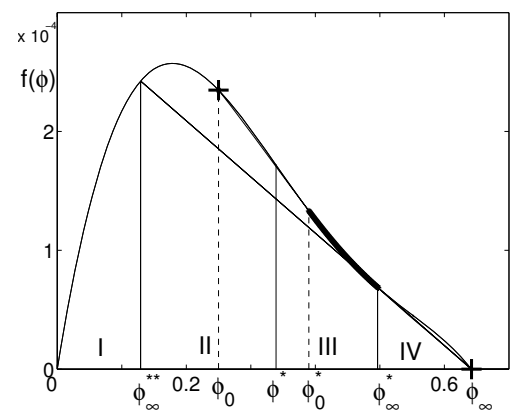

(b) fronts, rarefaction and constant areas

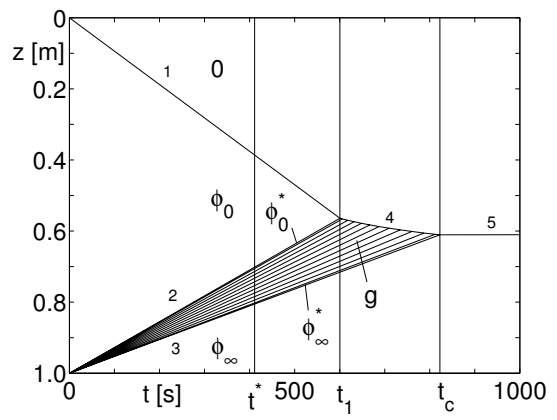

(c) profile at $t^{*}=t_{\mathrm{c}} / 2$

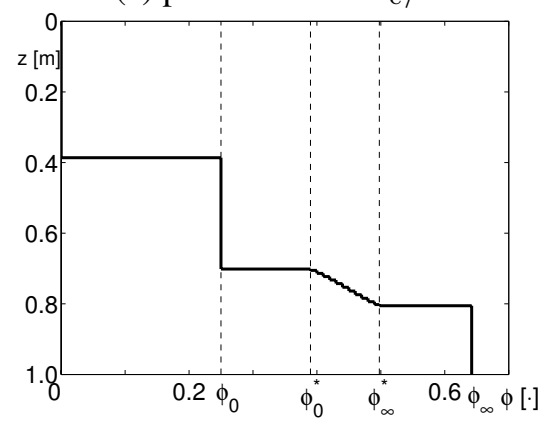

Fig. 5 Example 1: (a) flux plot, after Shannon et al. [48], (b) global solution, (c) profile.

Table 2 Example 1: Regions I-IV of different solutions to $\phi_{t}+f(\phi)_{z}=0$.

\begin{tabular}{lcccc}
\hline & Region I & Region II & Region III & Region IV \\
\hline$\phi_{0}$-Interval & {$\left[0, \phi_{\infty}^{* *}\right)$} & {$\left[\phi_{\infty}^{* *},<\phi^{*}\right)$} & {$\left[\phi^{*}, \phi_{\infty}^{*}\right)$} & {$\left[\phi_{\infty}^{*}, \phi_{\infty}\right]$} \\
Transition $\phi_{0} \rightarrow \phi_{\infty}$ & $\mathrm{S}$ & SRS & RS & S \\
Mode of Sedimentation [14] & MS-1 & MS-4 & MS-5 & MS-1 \\
\hline
\end{tabular}

structure of the initial value problem in dependence of the choice of the chosen initial concentration $\phi_{0}$. In Example 1, we use the flux function by Shannon et al. [48] given by $f(\phi)=\chi_{[0,0.642]}(\phi) \tilde{f}(\phi)$, where

$$
\tilde{f}(\phi)=\left(0.33843 \phi-1.37672 \phi^{2}+1.62275 \phi^{3}+0.11264 \phi^{4}-0.902253 \phi^{5}\right) \times 10^{-2} \mathrm{~m} / \mathrm{s} .
$$

Solutions of (2.1), (2.8) with a flux function that has a finite number of inflection points can be classified geometrically. Any two states $\phi^{-}, \phi^{+} \in\left[0, \phi_{\infty}\right]$ that satisfy the Rankine-Hugoniot condition (3.4) are said to satisfy the Oleĭnik entropy condition if

$$
\sigma\left(\phi^{-}, \phi^{+}\right) \leq \sigma\left(\phi^{-}, \phi\right) \text { for all } \phi \in\left[\phi^{-}, \phi^{+}\right] \cup\left[\phi^{+}, \phi^{-}\right]
$$

We discuss this classification for the particular flux function $f(\phi)$ given here, see Fig. 5. While $\phi^{-}=0$ can be connected to each state $\phi^{+} \in\left(0, \phi_{\infty}\right]$ by a shock satisfying the Oleĭnik entropy condition, the connection of a state $\phi^{-} \in\left[0, \phi_{\infty}\right]$ to $\phi^{+}=\phi_{\infty}$ depends on the location of $\phi^{-}$. If we define $\phi^{*}$ as the smaller inflection point and $\phi_{\infty}^{*}$ such that the line between $\left(\phi_{\infty}^{*}, f\left(\phi_{\infty}^{*}\right)\right)$ and $\left(\phi_{\infty}, f\left(\phi_{\infty}\right)\right)$ in the flux plot is tangent to the graph of the flux function and the state $\left(\phi_{\infty}^{* *}, f\left(\phi_{\infty}^{* *}\right)\right), \phi_{\infty}^{* *}<\phi_{\infty}^{*}$ is on the intersection of this tangent with the graph of the flux function,

$$
f\left(\phi_{\infty}^{*}\right)=f^{\prime}\left(\phi_{\infty}^{*}\right)\left(\phi_{\infty}^{*}-\phi_{\infty}\right), \quad f\left(\phi_{\infty}^{* *}\right)=f^{\prime}\left(\phi_{\infty}^{*}\right)\left(\phi_{\infty}^{* *}-\phi_{\infty}\right), \quad \phi_{\infty}^{* *}<\phi_{\infty}^{*},
$$

then $\left[0, \phi_{\infty}\right]$ can be divided into four regions (subintervals) as indicated in Table 2.

For instance, a state $\phi_{0}$, which is taken from region II, is connected to a state $\phi_{0}^{*}$ by a shock, between $\phi_{0}^{*}$ and $\phi_{\infty}^{*}$ the slope increases, which leads to a rarefaction, and $\phi_{\infty}^{*}$ is connected to $\phi_{\infty}$ by a shock,

$$
\begin{aligned}
& f\left(\phi_{0}\right)-f\left(\phi_{0}^{*}\right)=f^{\prime}\left(\phi_{0}^{*}\right)\left(\phi_{0}-\phi_{0}^{*}\right), \quad f\left(\phi_{\infty}^{*}\right)=f^{\prime}\left(\phi_{\infty}^{*}\right)\left(\phi_{\infty}^{*}-\phi_{\infty}\right), \\
& \phi^{-}, \phi^{+} \in\left(\phi_{0}^{*}, \phi_{\infty}^{*}\right), \quad \phi^{-}<\phi^{+} \Rightarrow f^{\prime}\left(\phi^{-}\right)<f^{\prime}\left(\phi^{+}\right) .
\end{aligned}
$$

For a flux density function with up to two inflection points, there are seven qualitatively different solutions (modes of sedimentation), numbered MS-1 to MS-7 in the notation of [14], to which we refer for details. The regions in the flux plot correspond to different modes, see Table 2.

For each choice of $\phi_{0}$, a global solution can be constructed either by the method of characteristics [14] or may be approximated by the front tracking method, as is demonstrated here for Example 1 with $\phi_{0}=0.25$. The structure of the global solution is shown in Fig. 5 (a): the shock $z_{1}$ emerges from $z=0$ and the contact discontinuities $z_{2}$ and $z_{3}$ emerge from $z=L$. They are given by the respective equations $z_{1}(t)=\sigma\left(0, \phi_{0}\right) t, z_{2}(t)=f^{\prime}\left(\phi_{0}^{*}\right) t+L$ and $z_{3}(t)=f^{\prime}\left(\phi_{\infty}^{*}\right) t+L$ for sufficiently small $t$. The discontinuities $z_{1}$ and $z_{2}$ intersect at a time $t_{1}$. Between $z_{2}$ and $z_{3}$, the solution undergoes a rarefaction wave and varies continuously as a function of $z / t$; for $0 \leq t \leq t_{1}$ and $z \in\left(z_{3}(t)\right.$, $\left.z_{2}(t)\right)$, it is given by 
$\phi(z, t)=g(z / t)$, where $g$ is the inverse of $f^{\prime}$ restricted to $\left[\phi_{0}^{*}, \phi_{\infty}^{*}\right]$. The merger of $z_{1}$ and $z_{2}$ produces a new discontinuity, $z_{4}$, whose trajectory is given by the initial value problem for an ordinary differential equation

$$
z_{4}\left(t_{1}\right)=z_{1}\left(t_{1}\right), \quad \dot{z}_{4}=\sigma\left(0, g\left(z_{4}(t) / t\right)\right), \quad t_{1} \leq t \leq t_{\mathrm{c}},
$$

where $t_{\mathrm{c}}$ is the time at which $z_{3}$ and $z_{4}$ meet. Finally, $z_{3}$ and $z_{4}$ merge to produce a stationary shock, $z_{5}(t)=z_{\mathrm{c}}$ for $t>t_{\mathrm{c}}$, which separates the regions of constancy $\phi(z, t)=0$ for $z \leq z_{5}(t)$ and $\phi(z, t)=\phi_{\infty}$ for $z>z_{5}(t)$.

\subsection{Free Riemann problem}

In Sect. 2 it was mentioned that either the sediment composition $\Phi_{\infty}$ appearing in the initial datum (2.8) could be prescribed explicitly, which gives rise to a Riemann problem between $\Phi_{0}$ and $\Phi_{\infty}$ posed at $t=0$, or one could simply specify that $\Phi_{\infty}$ is some state on the maximum packing manifold $\partial^{\infty}$. We address the latter case as a free Riemann problem, where the right state is not fixed but is supposed to belong to the manifold $\partial^{\infty}$. The solution construction shows that this is still a well-defined initial condition, since the sediment state $\Phi_{\infty}$ is selected as the intersection between the elementary curve of the first characteristic family with $\partial^{\infty}$, which leads to a solution that satisfies the Liu entropy condition. Moreover, this solution of the free Riemann problem is equivalent to the solution of the Riemann problem where the right state is fixed to be any state $\Phi_{\infty}^{\text {fixed }}$ on $\partial^{\infty}$. The intersecting state $\Phi_{\infty}$ is connected to $\Phi_{\infty}^{\text {fixed }}$ by a jump of zero speed. The discontinuity of the flux function due to the cut-off of the hindered settling function (see Sect. 2) induces a discontinuity of the Hugoniot locus, which may exclude existence of a solution.

\subsection{Example 2: Sedimentation of a bidisperse suspension}

Schneider et al. [47] report a settling experiment with a bidisperse suspension $(N=2)$ of particles with $d_{1}=4.96 \times 10^{-4} \mathrm{~m}$ and $d_{2}=1.25 \times 10^{-4} \mathrm{~m}$ (such that $\delta_{2}=0.06351$ ) and the common density $\varrho_{\mathrm{s}}=2790 \mathrm{~kg} / \mathrm{m}^{3}$, which are allowed to settle in a viscous fluid of density $\varrho_{\mathrm{f}}=1208 \mathrm{~kg} / \mathrm{m}^{3}$ and viscosity $\mu_{\mathrm{f}}=0.02416 \mathrm{~Pa} \mathrm{~s}$. In the terminology of the MLB model, the data used correspond to a flux function given by (2.9), where $V(\phi)$ is defined by (2.5) with $C=4.7$ and $\mu=8.78 \times 10^{-3} \mathrm{~m} / \mathrm{s}$. The settling column has height $L=0.3 \mathrm{~m}$. The initial concentration is $\Phi_{0}=(0.2,0.05)^{\mathrm{T}}$.

(a)

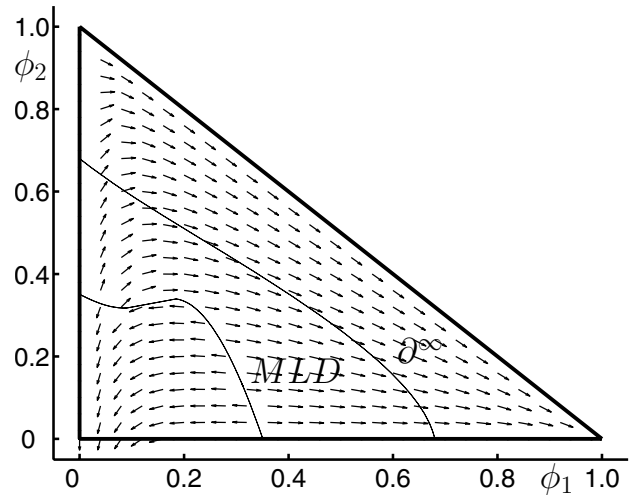

(b)

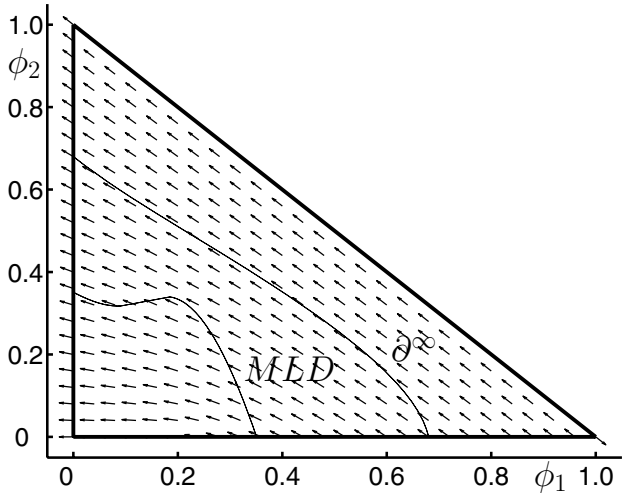

Fig. 6 Example 2: Right eigenvectors of $\mathcal{J}_{f}(\Phi)$ corresponding to (a) small and (b) large eigenvalues

In Fig. 6, the eigenvectors corresponding to the small and large eigenvalues of the flux Jacobian are depicted such that they point into the direction of increasing eigenvalues. The first characteristic family contains a MLD at which the eigenvalues assume a minimum with respect to the rarefaction curves. The second characteristic family is genuinely nonlinear.

In [47], also the underlying initial value problem (2.1), (2.8), where $\Phi_{\infty} \in \partial^{\infty}$, is implicitly defined as a state located on the maximum packing boundary (2.6), (2.7), is solved by constructing kinematic shocks, where all transitions in phase space are resolved by jumps. Therefore, the Liu entropy condition, which is the generalization of the Olernik entropy condition to systems, is not obeyed for some discontinuities. The main improvement of this work is that the Liu entropy condition is regarded.

A jump connecting $\Phi_{0}$ to $\Phi_{\infty} \in \partial^{\infty}$ violates the Liu entropy condition (3.6). This can be shown numerically by a counterexample. According to (3.6), a shock connecting $\Phi_{0}$ with $\Phi_{\infty}$ is only admissible if $\sigma\left(\Phi_{0}, \Phi_{\infty}\right) \leq \sigma\left(\Phi_{0}, \Phi\right)$ for all $\Phi$ on the Hugoniot locus of $\Phi^{0}$ in direction to $\Phi_{\infty}$. The state $\Phi_{\infty}=(0.6724,0.0303)^{\mathrm{T}}$ marks the intersection of the Hugoniot locus of $\Phi_{0}$ with $\partial^{\infty}$. We have $\sigma\left(\Phi_{0}, \Phi_{\infty}\right)=-1.020 \times 10^{-3} \mathrm{~m} / \mathrm{s}$. On the other hand, $\Phi_{0}^{*}=(0.4205,0.0448)^{\mathrm{T}}$ is that value on the Hugoniot locus of $\Phi_{0}$ which satisfies $f\left(\Phi_{0}\right)-f\left(\Phi_{0}^{*}\right)=\sigma\left(\Phi_{0}, \Phi_{0}^{*}\right)\left(\Phi^{0}-\Phi_{0}^{*}\right)$ where $\sigma\left(\Phi_{0}, \Phi_{0}^{*}\right)=\lambda\left(\Phi_{0}^{*}\right)$. 
We have the shock speed $\sigma\left(\Phi_{0}, \Phi_{0}^{*}\right)=-1.24 \times 10^{-3} \mathrm{~m} / \mathrm{s}$. Comparing the computed shock speeds we see that the Liu entropy condition is violated for a shock that connects $\Phi_{0}$ to $\Phi_{\infty}$.

\subsection{Modes of sedimentation in phase space}

In this section, regions in the phase space (of concentrations) are detected which generalize the intervals I-IV in the scalar flux plot. An initial value taken from one of those regions determines the mode of sedimentation, which classifies the solution of the initial value problem. For scalar equations, the mode of sedimentation can immediately be read off from the flux plot [14]. For systems, the mode depends on how the convex hull between the initial value and the maximum packing concentration is related to the flux plot. The Riemann problem is solved by using only the first characteristic family, where the state $\Phi_{\infty}$ is implicitly defined as the state at the intersection of the maximum packing boundary and the first characteristic family. Whereas this intersection is unique, the discontinuity of the flux function allows general non-unique solutions of Riemann problems (including the first and second characteristic family).

In order to collect states by regions which correspond to a mode, one constructs backward elementary curves from values on $\partial^{\infty}$. On the $\phi_{1}$ - and $\phi_{2}$-axes, the situation simplifies to that of a scalar equation. By the smoothness of the eigensystem, the backward construction can certainly also be done near the axes. There is no certainty whether the same modes can always be identified for general systems of equations.

But there is numerical evidence that the same backward construction can be done in an analogous way for the regions in phase space as for the intervals I-IV which can be identified for the scalar flux function in Fig. 5 (a). The intervals on the real axis correspond to regions in the phase space.

(a)

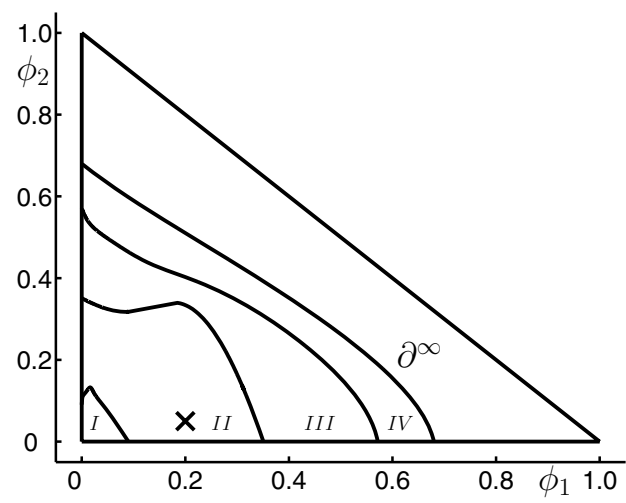

(b)

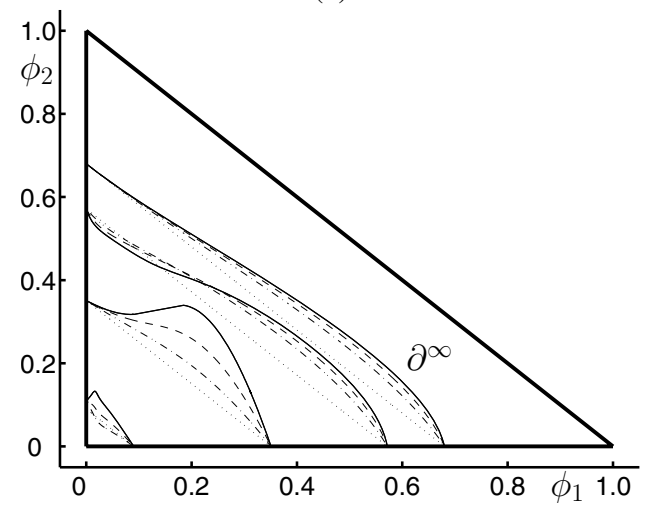

Fig. 7 Example 2: Concentration triangle with maximum packing due to Jeschar: (a) Modes of sedimentation modes for parameters from [47] and (b) for varying $\delta_{2}:=d_{2}^{2} / d_{1}^{2}$, namely: $\delta_{2}=1$ (dotted), $\delta_{2}=0.2$ (dash-dotted), $\delta_{2}=0.1$ (dashed), $\delta_{2}=0.0635$ (solid).

The classification of the modes for the second example is depicted in Fig. 7, where also a variation over $\delta_{2}$ indicates that for $\delta_{2} \approx 1$ the same classification holds. A general algebraic classification for arbitrary $\delta_{2}$ or $n$, or even an arbitrary hindrance function $V(\phi)$ is not available.

Values close to a state $\Phi_{\infty} \in \partial^{\infty}$ can be connected by a shock and thus lie in region IV. Each initial state from region IV can be connected to a state on $\partial^{\infty}$ by a shock which satisfies the Liu entropy condition. Note that at the boundary of the domain we have $f\left(\Phi_{\infty}\right)=0$ due to the cut-off of the flux function.

The boundary between regions III and IV is the set of all states $\Phi_{\infty}^{*}$ which can be connected to a state $\Phi_{\infty} \in \partial^{\infty}$ satisfying the tangent condition

$$
f\left(\Phi_{\infty}^{*}\right)-f\left(\Phi_{\infty}\right)=\sigma\left(\Phi_{\infty}^{*}, \Phi_{\infty}\right)\left(\Phi_{\infty}^{*}-\Phi_{\infty}\right), \quad \sigma\left(\Phi_{\infty}^{*}, \Phi_{\infty}\right)=\lambda_{1}\left(\Phi_{\infty}^{*}\right) .
$$

The boundary between region II and III appears to be a MLD, which is characterized as $r_{1}(\Phi) \cdot \nabla \lambda_{1}(\Phi)=0$.

Each initial state from region III can be connected to a state of the boundary III-IV by a rarefaction curve, and the respective boundary state is connected to a state on $\partial^{\infty}$. Finally, the boundary I-II is characterized as

$$
f(\Phi)-f\left(\Phi_{\infty}\right)=\sigma\left(\Phi_{\infty}^{*}, \Phi_{\infty}\right)\left(\Phi-\Phi_{\infty}\right), \quad \lambda_{1}(\Phi) \neq \sigma\left(\Phi_{\infty}^{*}, \Phi_{\infty}\right)
$$

where the pair of maximum packing $\Phi_{\infty}$ and tangent state $\Phi_{\infty}^{*}$, and thus the speed $\sigma\left(\Phi_{\infty}^{*}, \Phi_{\infty}\right)$ is taken from the previous calculation in (5.1). Each initial state from region II can be connected to a state $\Phi_{0}^{*}$ in region III by a shock whose speed 

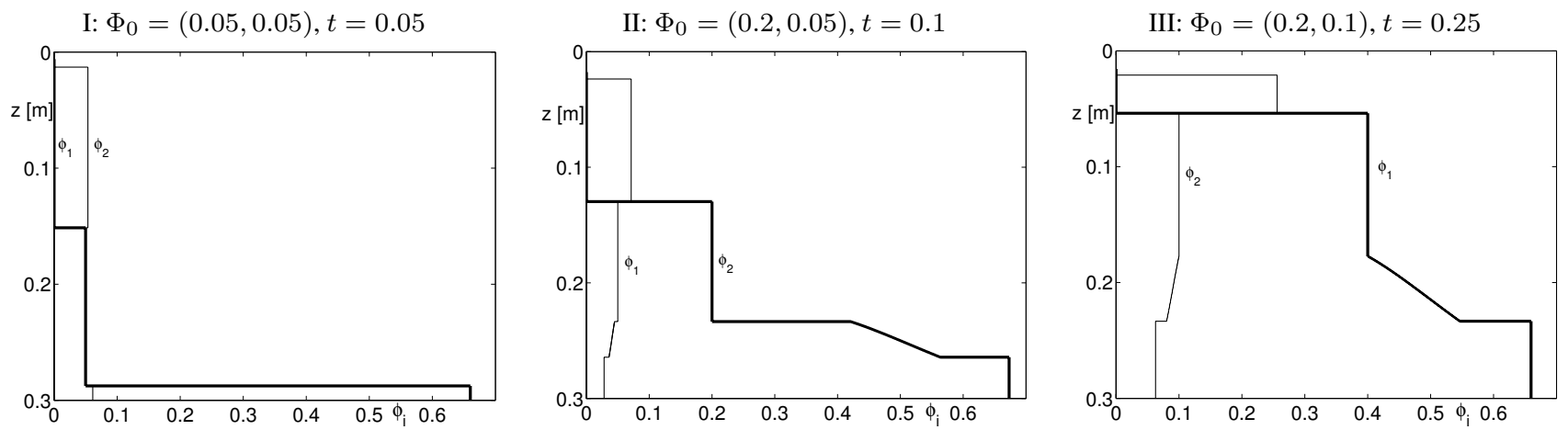

Fig. 8 Example 2: Profiles for $\Phi_{0}$ from regions I-III.

(a)

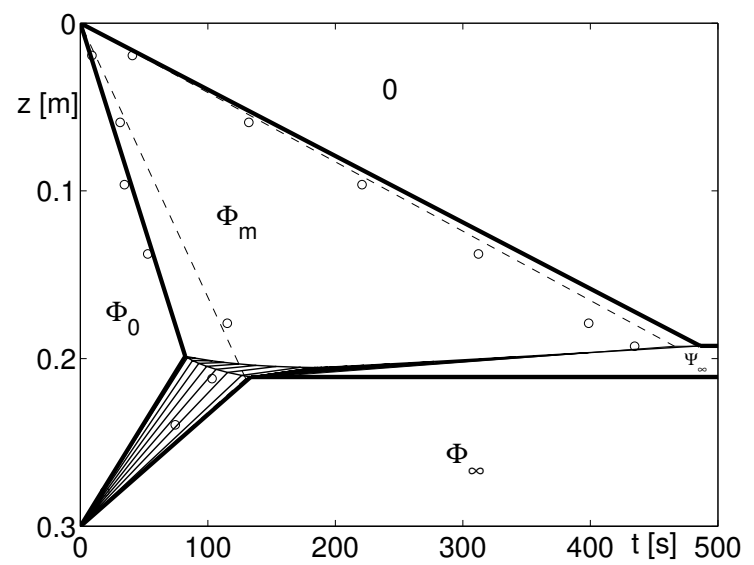

(b)

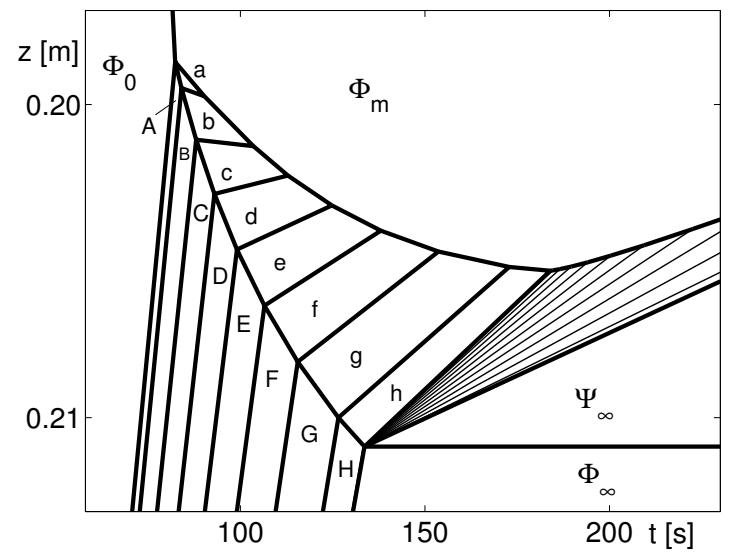

Fig. 9 Example 2: Calculated fronts for the discretization $\delta=0.02$ (a) comparison with experimental data (circles) and kinematic shock calculation (dashed lines), both taken from [47], (b) zoom.

equals $\lambda_{1}\left(\Phi_{0}^{*}\right)$. This state in region III in turn is connected to a state of $\partial^{\infty}$ as described before. Each initial state from region I can be connected to a state on $\partial^{\infty}$ by a shock satisfying the Liu entropy condition.

The richest solution structure is obtained for initial data which lead to mode II sedimentation, whereas the solution structures of all other modes can be considered as simpler variants. For illustration, the profiles of the modes which correspond to initial values taken from regions I, II, and III are depicted in Fig. 8. The profiles are generated by the initialization of the front tracking algorithm, which is explained in Sect. 5.5. The resolution $\delta=0.001$ determines the sampling density of rarefaction curves at the approximate solution of Riemann problems in phase space.

\subsection{Front tracking method}

The initial value problem for the following $2 \times 2$-system of conservation laws, together with the initial datum (2.8), is solved by the front tracking method:

$$
\Phi_{t}+f(\Phi)_{x}=0, \quad \Phi \in \mathbb{R}^{2}, \quad x \in \mathbb{R}, \quad f \in C^{2}, \quad t>0 .
$$

The principle of the front tracking method is to approximate the initial data by a piecewise constant function and to solve the Riemann problems approximately at each jump in those data. In the $x$ - $t$ diagram, the wave fronts between two neighboring Riemann problems may collide, generating a new Riemann problem at collision time, which in turn creates a new fan of wave fronts [22]. For a scalar flux function the approximation of the Riemann problem can be considered as a result of the approximation of the flux function by a polygon [13, 15, 21]. For systems (with vector-valued flux functions), the Riemann problem is approximated by picking a finite number of states in the phase space on the rarefaction segments of the exact solution to the Riemann problem.

In order to avoid a blow-up of the number of fronts, so-called weak waves need to be eliminated [44]: To identify weak waves, all fronts are labeled with a "generation" [22, pp. 218ff.]. At the initial time the generations are set to zero. Assume 
(a) $t=100$

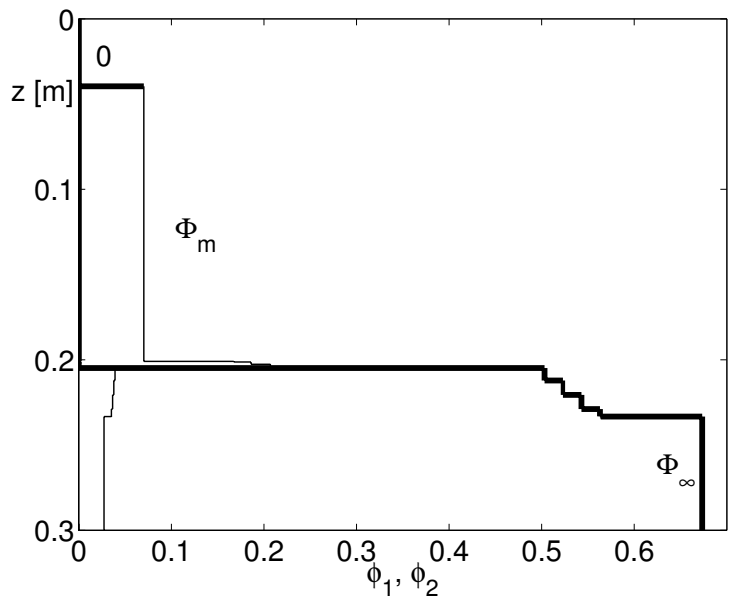

(b) $t=200$

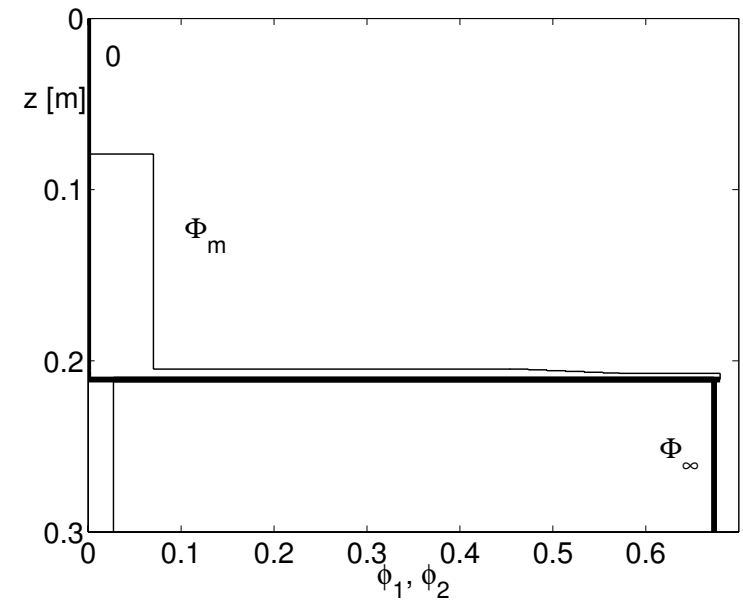

Fig. 10 Example 2: Profiles at two different times.

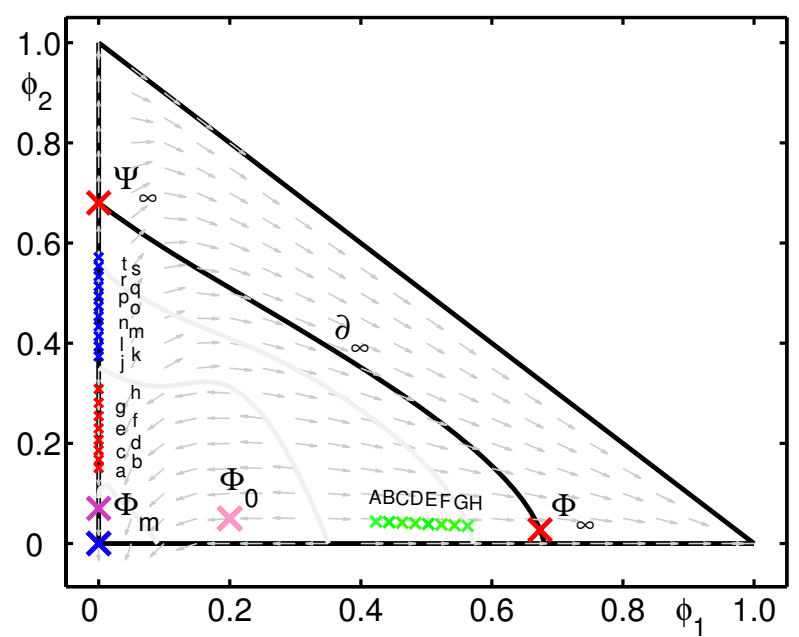

Fig. 11 (online colour at: www.zamm-journal.org) Example 2: States assumed during the front tracking solution of (5.2), (2.8).

that two fronts having generation $m$ and $n$ belonging to two different families collide. Then the generation of the resulting fronts which belong to the same family are assigned the generation numbers $m$ and $n$, respectively. The generation of the other fronts, however, is set to $n+m$. Thus, fronts of higher generation are obtained which are weak in the sense that they have negligible impact on the solution.

The approximate solution $\Phi^{\delta}$ converges to the entropy solution as $\delta \rightarrow 0$. The following algorithmic description of the front tracking method, which is applied to approximate the solution of a one-dimensional strictly hyperbolic system of conservation laws, $\Phi_{t}+f(\Phi)_{x}=0,\left.\Phi\right|_{t=0}=\Phi_{0}$, is taken from [22]:

1. Approximate initial data $\Phi_{0}$ by a piecewise constant function $\Phi_{0}^{\delta}$.

2. Approximate the solution of each Riemann problem by a piecewise constant function by sampling points with distance $\delta$ on the rarefaction curve, using exact shocks and contact discontinuities and eliminating weak waves.

3. Track discontinuities ("fronts").

4. Continue to solve Riemann problems approximately as in (2).

This front tracking algorithm relies on the solution of Riemann problems, which is done here by the concatenation of elementary waves (4.1). The computation of elementary waves is described in Algorithm 4.1.

\subsection{Example 2 (continued): comparison with results by Schneider et al. [47]}

For illustration, the solution of the initial value problem (2.8), (5.2) with $\Phi_{0}=(0.2,0.05)^{\mathrm{T}}$ (Example 2) is generated by the front tracking method with $\delta=0.02$. The fronts generated are shown in a $z$-t-plot in Fig. 9. It can be clearly recognized that there is a centered rarefaction wave originating from the vessel bottom. This rarefaction refines the kinematic shock 
Table 3 Example 2: States assumed during the front tracking solution of (5.2), (2.8).

\begin{tabular}{lcccccccc}
\hline & $\phi_{1}$ & $\phi_{2}$ & & $\phi_{1}$ & $\phi_{2}$ & & $\phi_{1}$ & $\phi_{2}$ \\
\hline $\mathbf{0}$ & 0 & 0 & $\Phi_{0}$ & 0.2000 & 0.0500 & $\Phi_{\mathrm{m}}$ & 0 & 0.0703 \\
$\Phi_{\infty}$ & 0.6734 & 0.0273 & $A$ & 0.4232 & 0.0439 & $B$ & 0.4432 & 0.0428 \\
$C$ & 0.4631 & 0.0416 & $D$ & 0.4831 & 0.0404 & $E$ & 0.5031 & 0.0391 \\
$F$ & 0.5230 & 0.0379 & $G$ & 0.5430 & 0.0366 & $H$ & 0.5629 & 0.0353 \\
$I$ & 0.5638 & 0.0352 & $a$ & 0 & 0.1510 & $b$ & 0 & 0.1674 \\
$c$ & 0 & 0.1860 & $d$ & 0 & 0.2070 & $e$ & 0 & 0.2299 \\
$f$ & 0 & 0.2547 & $g$ & 0 & 0.2809 & $h$ & 0 & 0.3083 \\
$j$ & 0 & 0.3736 & $k$ & 0 & 0.3936 & $l$ & 0 & 0.4136 \\
$m$ & 0 & 0.4336 & $n$ & 0 & 0.4536 & $o$ & 0 & 0.4736 \\
$p$ & 0 & 0.4936 & $q$ & 0 & 0.5136 & $r$ & 0 & 0.5336 \\
$s$ & 0 & 0.5536 & $t$ & 0 & 0.5715 & $\Psi_{\infty}$ & 0 & 0.6800 \\
\hline
\end{tabular}

Table 4 Example 2: Riemann problems solved during the front tracking solution of (5.2), (2.8).

\begin{tabular}{cccclccccc}
\hline$\#$ & type & $u_{\mathrm{L}}$ & $u_{\mathrm{R}}$ & $U$ & $\#$ & type & $u_{\mathrm{L}}$ & $u_{\mathrm{R}}$ & $U$ \\
\hline 1 & 1 & 0 & $\Phi_{0}$ & $\Phi_{\mathrm{m}}$ & 17 & 4 & $\Phi_{\mathrm{m}}$ & $f$ & $\varnothing$ \\
2 & 2 & $\Phi_{0}$ & $\Phi_{\infty}$ & $A, B, C, D, E, F, G, H, I$ & 18 & 4 & $\Phi_{\mathrm{m}}$ & $g$ & $\varnothing$ \\
3 & 3 & $\Phi_{\mathrm{m}}$ & $A$ & $a$ & 19 & 4 & $\Phi_{\mathrm{m}}$ & $h$ & $\varnothing$ \\
4 & 3 & $a$ & $B$ & $b$ & 20 & 4 & $\Phi_{\mathrm{m}}$ & $j$ & $\varnothing$ \\
5 & 3 & $b$ & $C$ & $c$ & 21 & 4 & $\Phi_{\mathrm{m}}$ & $k$ & $\varnothing$ \\
6 & 4 & $\Phi_{\mathrm{m}}$ & $b$ & $\varnothing$ & 22 & 4 & $\Phi_{\mathrm{m}}$ & $l$ & $\varnothing$ \\
7 & 3 & $c$ & $D$ & $d$ & 23 & 4 & $\Phi_{\mathrm{m}}$ & $m$ & $\varnothing$ \\
8 & 3 & $d$ & $E$ & $e$ & 24 & 4 & $\Phi_{\mathrm{m}}$ & $n$ & $\varnothing$ \\
9 & 4 & $\Phi_{\mathrm{m}}$ & $c$ & $\varnothing$ & 25 & 4 & $\Phi_{\mathrm{m}}$ & $o$ & $\varnothing$ \\
10 & 3 & $e$ & $F$ & $f$ & 26 & 4 & $\Phi_{\mathrm{m}}$ & $p$ & $\varnothing$ \\
11 & 4 & $\Phi_{\mathrm{m}}$ & $d$ & $\varnothing$ & 27 & 4 & $\Phi_{\mathrm{m}}$ & $q$ & $\varnothing$ \\
12 & 3 & $f$ & $G$ & $g$ & 28 & 4 & $\Phi_{\mathrm{m}}$ & $r$ & $\varnothing$ \\
13 & 4 & $\Phi_{\mathrm{m}}$ & $e$ & $\varnothing$ & 29 & 4 & $\Phi_{\mathrm{m}}$ & $s$ & $\varnothing$ \\
14 & 3 & $g$ & $H$ & $h$ & 30 & 4 & $\Phi_{\mathrm{m}}$ & $t$ & $\varnothing$ \\
15 & 4 & $h$ & $I$ & $\varnothing$ & 31 & 4 & $\Phi_{\mathrm{m}}$ & $\Psi_{\infty}$ & $\varnothing$ \\
16 & 5 & $h$ & $\Phi_{\infty}$ & $j, k, l, m, n, o, p, q, r, s, t, \Psi_{\infty}$ & 32 & 4 & 0 & $\Psi_{\infty}$ & $\varnothing$ \\
\hline
\end{tabular}

construction made in [47] (dashed lines; the circles correspond to the experimental values) and establishes the analogy to the scalar equation. The dashed lines delimiting the sediments are not visible since they lie on the solid lines namely those representing the lower bound of the rarefaction fan and the stationary sediment. The deviation of the visible dashed lines is caused by a rounding error in the last digits, and thus may serve as an indication of the strong sensitivity of the solution on the initial data. Note that the rarefaction wave is in accordance with the entropy condition, while a single discontinuity violates the Liu entropy condition. The rarefaction feature has also been observed in previous numerical simulations of the same problem by finite difference methods $[9,10]$.

This smooth concentration transition describes the gradual decrease of settling velocity at higher concentrations. It has to be observed that this smooth transition occurs for both species simultaneously. The rarefaction interacts with the upper interface, describing a mechanism which builds a second sediment layer. This mechanism has no analogue in the scalar case. The profiles at two different times are displayed in Fig. 10. The states assumed are listed in Table 3, see also Fig. 11. The Riemann problems that are solved are listed in Table 4 in chronological order. We use the notation $R P_{i}\left(u_{\mathrm{L}}, u_{\mathrm{R}}\right)=U$ to express that $u_{\mathrm{L}}, u_{\mathrm{R}}$ are the left and right states of a Riemann problem of type $i, i=1, \ldots, 5$, and where $U$ contains the resulting intermediate states of the approximate solution. The result $\varnothing$ means that the solution of a Riemann problem consists in one single shock only. The five different types of Riemann problem correspond to the classification of Sect. 5.7. 
(a) $\Phi_{0}=(0.05,0.05)^{\mathrm{T}}$

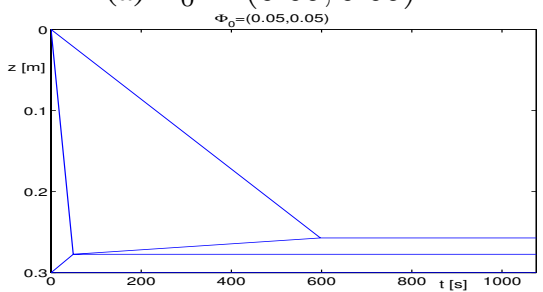

(d) $\Phi_{0}=(0.2,0.3)^{\mathrm{T}}$

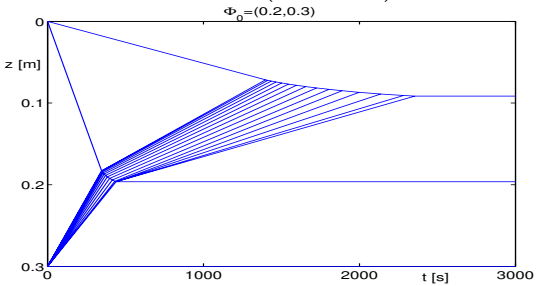

(b) $\Phi_{0}=(0.002,0.1)^{\mathrm{T}}$

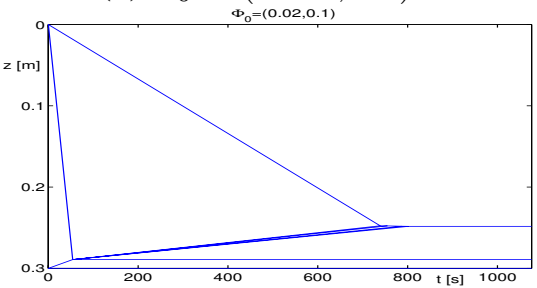

(e) $\Phi_{0}=(0.1,0.4)^{\mathrm{T}}$

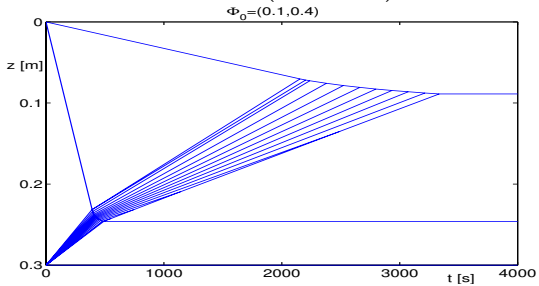

(c) $\Phi_{0}=(0.2,0.1)^{\mathrm{T}}$

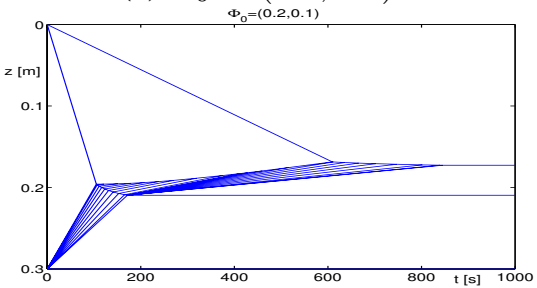

(f) $\Phi_{0}=(0.4,0.1)^{\mathrm{T}}$

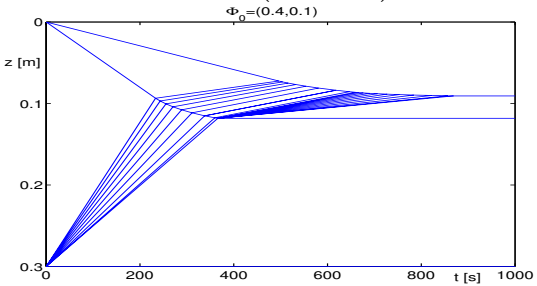

Fig. 12 (online colour at: www.zamm-journal.org) Example 2: Fronts created during a run with varying initial data and discretization $\delta=0.02$.

Various solutions which are obtained by applying the front tracking algorithm to the initial value problem with diverse initial data, are shown in Fig. 12.

\subsection{Classification of Riemann problems}

In this section, the Riemann problems, which have to be solved when performing the front tracking algorithm for the second example, are classified. Here, $\Phi_{0} \in \mathcal{D}$ is an initial value and $\Phi_{\infty} \in \partial^{\infty}$ is a boundary value on $\partial^{\infty}$. At initial time, two Riemann problems need to be solved, one for the suspension-supernate interface and one for the sediment-suspension interface, where the solution depends on the sedimentation mode. The two resulting wave fans interact, giving a stationary profile after finite time. The special feature of this initial value problem is that the 1-wave becomes scalar whenever the left state is on the $\phi_{2}$-axis. If, moreover, also the right state is on the $\phi_{2}$-axis, the whole Riemann problem to solve becomes scalar. Altogether, the following types of Riemann problems arise:

Type 1. The solution of $R P_{1}\left(\mathbf{0}, \Phi_{0}\right)$ consists of two shocks. The 1-shock connects the state $\mathbf{0}$ to some intermediate state $\Phi_{\mathrm{m}}$ on the $\phi_{2}$-axis by a front of speed $\sigma\left(\mathbf{0}, \Phi_{\mathrm{m}}\right)=f_{2}\left(\Phi_{\mathrm{m}}\right) / \phi_{\mathrm{m} 2}$. The 2-shock connects $\Phi_{0}$ to an intermediate state $\Phi_{\mathrm{m}}=\left(0, \phi_{\mathrm{m} 2}\right)$ that satisfies $f\left(\Phi_{\mathrm{m}}\right)-f\left(\Phi_{0}\right)=\sigma\left(\Phi_{\mathrm{m}}, \Phi_{0}\right)\left(\Phi_{\mathrm{m}}-\Phi_{0}\right)$.

Type 2. We here deal with a free Riemann problem, see Sect. 5.2. The solution of $R P_{2}\left(\Phi_{0}, \Phi_{\infty}\right)$ consists only of a 1-wave, which is constructed along the lines of Sect. 3, with the modification that the condition $\Phi_{\infty} \in \partial^{\infty}$ has to be added in order to specify the valid maximum packing density. The solution of $R P_{2}\left(\Phi_{0}, \Phi_{\infty}\right)$ depends on the phase space region in which $\Phi_{0}$ is located.

I \& IV. If $\Phi_{0}$ is located in region I or IV, then the solution of $R P_{2}\left(\Phi_{0}, \Phi_{\infty}\right)$ consists of a shock connecting $\Phi_{0}$ to $\Phi_{\infty}$ by satisfying $\Phi_{\infty} \in \partial^{\infty}$ together with $f\left(\Phi_{0}, \Phi_{\infty}\right)=\sigma\left(\Phi_{0}, \Phi_{\infty}\right)\left(\Phi_{0}-\Phi_{\infty}\right)$.

II. If $\Phi_{0}$ is located in region II, then the solution of $R P_{2}\left(\Phi_{0}, \Phi_{\infty}\right)$ is a double-sided contact wave, which consists of a shock connecting $\Phi_{0}$ to $\Phi_{0}^{*}$, where $f\left(\Phi_{0}\right)-f\left(\Phi_{0}^{*}\right)=\sigma\left(\Phi_{0}, \Phi_{0}^{*}\right)\left(\Phi_{0}-\Phi_{0}^{*}\right)$, a rarefaction wave connecting $\Phi_{0}^{*}$ to $\Phi_{\infty}^{*}$, and, finally, a shock connecting $\Phi_{\infty}^{*}$ to $\Phi_{\infty}$ that satisfies $f\left(\Phi_{\infty}^{*}\right)-f\left(\Phi_{\infty}\right)=\sigma\left(\Phi_{\infty}^{*}, \Phi_{\infty}\right)\left(\Phi_{\infty}^{*}-\Phi_{\infty}\right)$, where $\Phi_{\infty}^{*}$ is on the rarefaction curve through $\Phi_{0}^{*}$ and $\Phi_{\infty}$ is constrained by $\Phi_{\infty} \in \partial^{\infty}$.

III. If $\Phi_{0}$ is located in region III, then the solution of $R P_{2}\left(\Phi_{0}, \Phi_{\infty}\right)$ consists of a contact wave, where a rarefaction between $\Phi_{0}$ and $\Phi_{\infty}^{*}$ is connected to a shock between $\Phi_{\infty}^{*}$ and $\Phi_{\infty}$ in the same way as in mode II.

Type 3. The Riemann problem $R P_{3}(\Psi, \Phi)$ with $\Psi=\left(0, \psi_{2}\right), \Phi=\left(\phi_{1}, \phi_{2}\right), \phi_{1}, \phi_{2} \neq 0$ is solved by a 1 -wave in scalar variables that has potentially all structures possible for a flux function with one inflection point. The 2-wave is a shock. All possible values for $\Phi$ in this Riemann problem are obtained from the solution of $R P_{2}\left(\Phi_{0}, \Phi_{\infty}\right)$ during the solution of the initial value problem under consideration.

Type 4. The Riemann problem $R P_{4}(\Psi, \Phi)$ with $\Psi=\left(0, \psi_{2}\right), \Phi=\left(0, \psi_{2}\right)$ is scalar and produces a 1-wave only.

Type 5. The solution of $R P_{5}\left(\Psi, \Phi_{\infty}\right)$ consists of a 1 -wave connecting $\Psi=\left(0, \psi_{2}\right)$ to $\Psi_{\infty}=\left(0, \phi_{\max }\right)$ having the same structure as in $R P_{2}$, followed by a shock of speed 0 , that connects $\Psi_{\infty}$ to $\Phi_{\infty}$. 
(a)

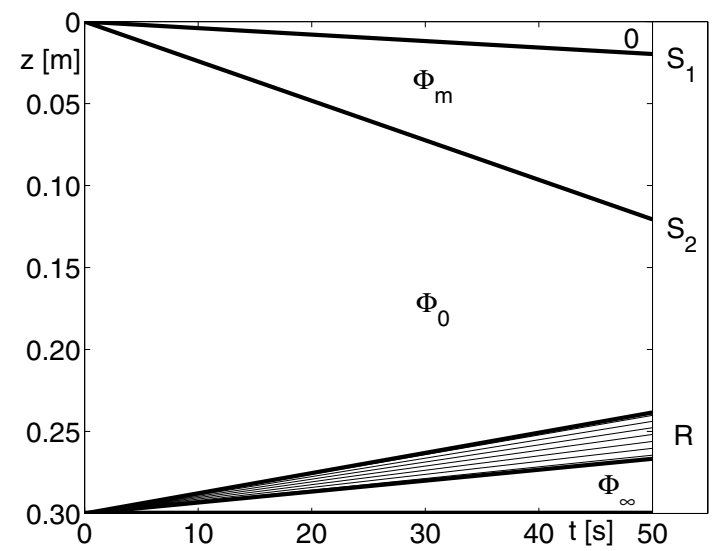

(c)

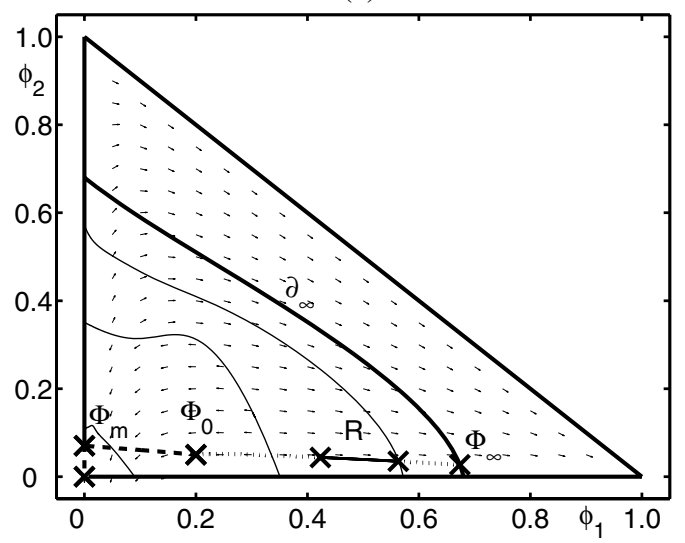

(b)

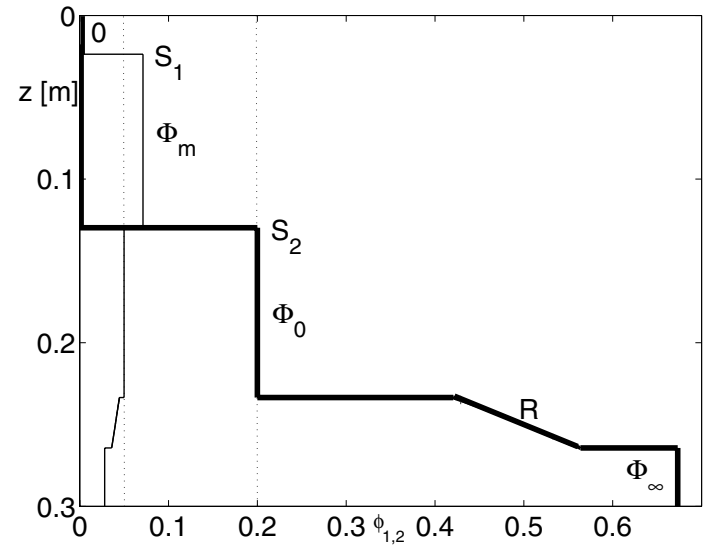

(d)

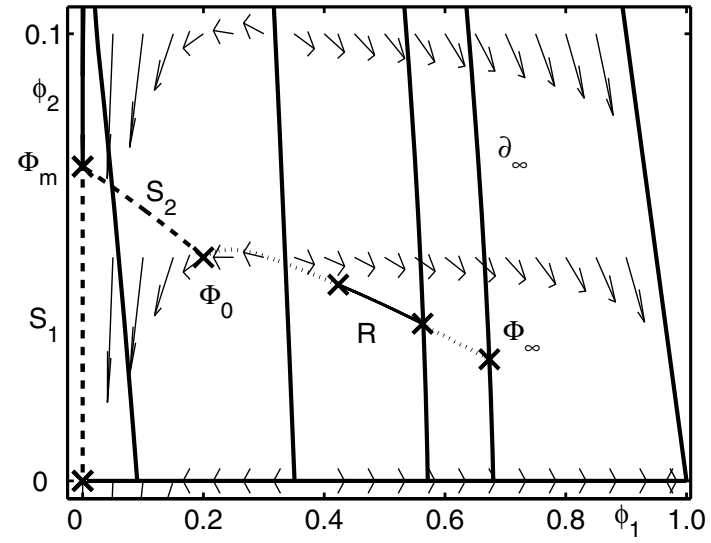

Fig. 13 Example 2: (a) Fronts created from initial data $\Phi_{0}=(0.2,0.05)^{\mathrm{T}}$, (b) profile at $t=50,(\mathrm{c}, \mathrm{d})$ states in phase space.

(a)

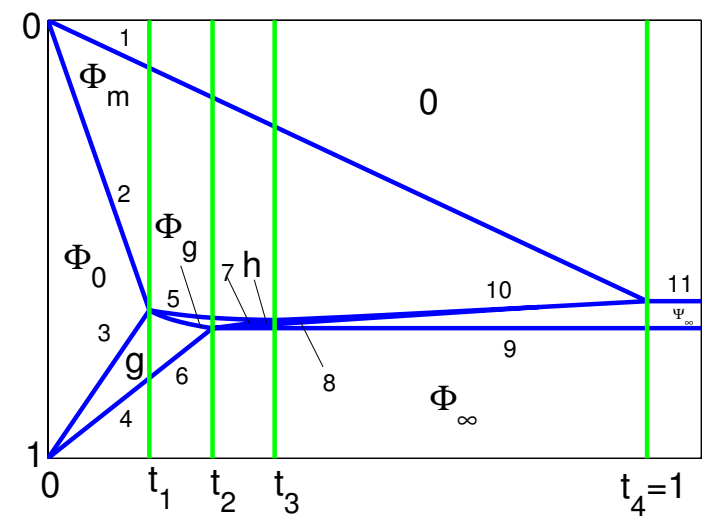

(b)

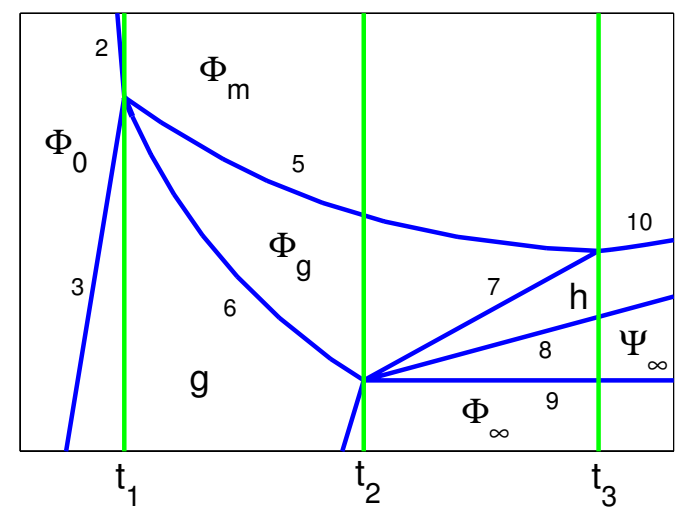

Fig. 14 (online colour at: www.zamm-journal.org) Example 2: Global solution.

\subsection{Description of the global solution}

The structure of the global solution and the possible construction by the method of characteristics, which is obtained by a reexamination of the solution by the front-tracking method, is displayed in Fig. 14. Compared with the front tracking or even the finite volume method, the method of characteristics is more accurate but therefore needs more prerequisites.

Since the initial value problem has piecewise constant initial data, the starting point for the method of characteristics is the same as in the front tracking method, namely the solution of the Riemann problems at the initial jumps. For the Riemann problem $\operatorname{RP}\left(0, \Phi_{0}\right)$ at the upper interface between the zero and initial concentrations, the intermediate state $\Phi_{\mathrm{m}}$ 
and the separating speeds $\sigma\left(\mathbf{0}, \Phi_{\mathrm{m}}\right), \sigma\left(\Phi_{0}, \Phi_{\mathrm{m}}\right)$ are calculated from the nonlinear system of equations

$$
f\left(\Phi_{\mathrm{m}}\right)=\sigma\left(\mathbf{0}, \Phi_{\mathrm{m}}\right) \Phi_{\mathrm{m}}, \quad f\left(\Phi_{0}\right)-f\left(\Phi_{\mathrm{m}}\right)=\sigma\left(\Phi_{0}, \Phi_{\mathrm{m}}\right)\left(\Phi_{0}-\Phi_{\mathrm{m}}\right) .
$$

For the solution of the Riemann problem $R P\left(\Phi_{0}, \Phi_{\infty}\right)$ it is crucial to satisfy the Liu entropy condition. The initial concentration $\Phi_{0}$ is connected to a state $\Phi_{0}^{*}$, and a concentration $\Phi_{\infty}$ on $\partial^{\infty}$ is connected to a state $\Phi_{\infty}^{*}$ by satisfying the following respective Rankine-Hugoniot conditions, where $\lambda_{1}(\Phi)$ is the smaller eigenvalue of $\mathcal{J}_{f}(\Phi)$ :

$$
\begin{aligned}
& f\left(\Phi_{0}\right)-f\left(\Phi_{0}^{*}\right)=\sigma\left(\Phi_{0}, \Phi_{0}^{*}\right)\left(\Phi_{0}-\Phi_{0}^{*}\right), \quad \lambda_{1}\left(\Phi_{0}^{*}\right)=\sigma\left(\Phi_{0}, \Phi_{0}^{*}\right), \\
& f\left(\Phi_{\infty}\right)-f\left(\Phi_{\infty}^{*}\right)=\sigma\left(\Phi_{\infty}, \Phi_{\infty}^{*}\right)\left(\Phi_{\infty}-\Phi_{\infty}^{*}\right), \quad \lambda_{1}\left(\Phi_{\infty}^{*}\right)=\sigma\left(\Phi_{\infty}, \Phi_{\infty}^{*}\right) .
\end{aligned}
$$

The state $\Phi_{0}^{*}$ is connected with $\Phi_{\infty}^{*}$ by a rarefaction curve $\mathcal{G}$, which is parametrized as

$$
\begin{aligned}
& \mathcal{G}\left(\Phi_{0}^{*}, 0\right)=\Phi_{0}^{*}, \quad \partial_{\tau} \mathcal{G}\left(\Phi_{0}^{*}, \tau\right)=r_{1}\left(\mathcal{G}\left(\Phi_{0}^{*}, \tau\right)\right), \quad \mathcal{G}\left(\Phi_{0}^{*}, \tau_{0}\right)=\Phi_{\infty}^{*}, \\
& r_{1}\left(\mathcal{G}\left(\Phi_{0}^{*}, \tau\right)\right) \cdot \nabla \lambda\left(\mathcal{G}\left(\Phi_{0}^{*}, \tau\right)\right)>0, \quad\left\|r_{1}\left(\mathcal{G}\left(\Phi_{0}^{*}, \tau\right)\right)\right\|_{2}=1 \quad \text { for } \tau \in\left[0, \tau_{0}\right] .
\end{aligned}
$$

From the parametrization of speed-state pairs

$$
G: \tau \mapsto(s, g)=\left(\lambda_{1}\left(\mathcal{G}\left(\Phi_{0}^{*}, \tau\right)\right), \mathcal{G}\left(\Phi_{0}^{*}, \tau\right)\right)
$$

one can extract the map $g: \mathbb{R} \rightarrow \mathbb{R}^{N}$ of speeds onto states in phase space, which corresponds to the inverse of the first derivative of the flux function for scalar governing equations.

For any solution containing jumps, the Liu entropy condition needs to be checked. For this purpose, the Hugoniot locus, e.g., between $\Phi_{\mathrm{m}}$ and $\Phi_{0}$, is described by

$$
\begin{aligned}
& \mathcal{H}\left(\Phi_{\mathrm{m}}, 0\right)=\Phi_{\mathrm{m}}, \quad \mathcal{H}\left(\Phi_{\mathrm{m}}, \tau_{\mathrm{m}}\right)=\Phi_{0}, \\
& f\left(\mathcal{H}\left(\Phi_{\mathrm{m}}, \tau\right)\right)-f\left(\Phi_{\mathrm{m}}\right)=\sigma\left(\mathcal{H}\left(\Phi_{\mathrm{m}}, \tau\right), \Phi_{\mathrm{m}}\right)\left(\mathcal{H}\left(\Phi_{\mathrm{m}}, \tau\right)-\Phi_{\mathrm{m}}\right) \quad \text { for } \tau \in\left[0, \tau_{\mathrm{m}}\right], \\
& \partial_{\tau} \mathcal{H}\left(\Phi_{\mathrm{m}}, 0\right)=r_{2}\left(\mathcal{H}\left(\Phi_{\mathrm{m}}, 0\right)\right), \quad \tau=\left\|\mathcal{H}\left(\Phi_{\mathrm{m}}, \tau\right)-\mathcal{H}\left(\Phi_{\mathrm{m}}, 0\right)\right\|_{2},
\end{aligned}
$$

such that speed-state pairs can be expressed as

$$
H_{i}: \tau \mapsto(s, h)=\left(\sigma\left(\Phi_{\mathrm{m}}, \mathcal{H}_{i}\left(\Phi_{\mathrm{m}}, \tau\right)\right), \mathcal{H}_{i}\left(\Phi_{\mathrm{m}}, \tau\right)\right)
$$

The fact that the Liu entropy condition $s\left(\tau_{\mathrm{m}}\right) \leq s(\tau)$ for all $\tau \in\left[0, \tau_{\mathrm{m}}\right]$ on the Hugoniot locus from $\Phi_{\mathrm{m}}$ (or any other state on the $\phi_{2}$-axis) to $\Phi_{0}$ (or any other state in $\mathcal{D}$ ) is satisfied can be read off from the eigenvector plot (Fig. 6) corresponding to the fast family, which indicates decreasing eigenvalues. The satisfaction of the Liu entropy condition needs to be verified for each Hugoniot locus, that is between $\mathbf{0}$ and $\Phi_{\mathrm{m}}, \Phi_{0}$ and $\Phi_{0}^{*}$, and $\Phi_{\infty}^{*}$ and $\Phi_{\infty}$.

In our case, the discontinuities $z_{1}(t)=\sigma\left(\mathbf{0}, \Phi_{\mathrm{m}}\right) t$ and $z_{2}(t)=\sigma\left(\Phi_{0}, \Phi_{\mathrm{m}}\right) t$ emerge from $z=0$, while $z_{3}(t)=$ $\sigma\left(\Phi_{0}, \Phi_{0}^{*}\right) t+L$ and $z_{4}(t)=\sigma\left(\Phi_{\infty}, \Phi_{\infty}^{*}\right) t+L$ emerge from $z=L$. If $z_{2}(t)$ and $z_{3}(t)$ collide at $t=t_{1}$ and we define $z_{\mathrm{c} 1}=z_{2}\left(t_{1}\right)=z_{3}\left(t_{1}\right)$, then the solution in $\left[0, t_{1}\right)$ can be stated as

$$
\Phi(z, t)= \begin{cases}0 & \text { for } z<z_{1}(t) \\ \Phi_{\mathrm{m}} & \text { for } z_{1}(t) \leq z<z_{2}(t) \\ \Phi_{0} & \text { for } z_{2}(t) \leq z<z_{3}(t), \quad 0 \leq t \leq t_{1} . \\ g(z / t) & \text { for } z_{3}(t) \leq z<z_{4}(t) \\ \Phi_{\infty} & \text { for } z \geq z_{4}(t)\end{cases}
$$

At the collision point $\left(z_{\mathrm{c} 1}, t_{1}\right)$, the curved shocks $z_{5}(t)$ and $z_{6}(t)>z_{5}(t)$ emerge. While the solution values immediately below the line $z_{6}$ follow from the application of $g$, those immediately above satisfy the jump condition

$$
f\left(\Phi_{\mathrm{g}}\left(z_{6}(t), t\right)\right)-f\left(g\left(z_{6}(t) / t\right)\right)=\sigma\left(\Phi_{\mathrm{g}}\left(z_{6}(t), t\right), g\left(z_{6}(t) / t\right)\right)\left(\Phi_{\mathrm{g}}\left(z_{6}(t), t\right)-g\left(z_{6}(t) / t\right)\right),
$$

and are propagated on characteristic curves as

$$
\Phi_{\mathrm{g}}(z, t)=\Phi_{\mathrm{g}}\left(z_{6}\left(t_{\mathrm{g}}\right), t_{\mathrm{g}}\right) \quad \text { if } z=z_{6}\left(t_{\mathrm{g}}\right)+\left(t-t_{\mathrm{g}}\right) \lambda_{1}\left(\Phi_{\mathrm{g}}\left(z_{6}\left(t_{\mathrm{g}}\right), t_{\mathrm{g}}\right)\right)
$$


for an appropriate $t_{\mathrm{g}} \in\left[t_{1}, t_{2}\right]$. The trajectories $z_{5}(t)$ and $z_{6}(t)$ are defined by

$$
z_{5}\left(t_{1}\right)=z_{\mathrm{c} 1}, \quad \dot{z}_{5}=\sigma\left(\Phi_{\mathrm{m}}, \Phi_{\mathrm{g}}\left(z_{5}(t), t\right)\right), \quad z_{6}\left(t_{1}\right)=z_{\mathrm{c} 1}, \quad \dot{z}_{6}=\sigma\left(\Phi_{\mathrm{g}}\left(z_{6}(t), t\right), g\left(z_{6}(t) / t\right)\right), \quad t>t_{1} .
$$

If we define $t_{2}>t_{1}$ to be the time at which $z_{4}(t)$ and $z_{6}(t)$ collide, with $z_{\mathrm{c} 2}=z_{6}\left(t_{2}\right)=z_{4}\left(t_{2}\right)$, then the solution in the time interval $\left[t_{1}, t_{2}\right)$ becomes

$$
\Phi(z, t)= \begin{cases}0 & \text { for } z<z_{1}(t) \\ \Phi_{\mathrm{m}} & \text { for } z_{1}(t) \leq z<z_{5}(t) \\ \Phi_{\mathrm{g}}(z, t) & \text { for } z_{5}(t) \leq z<z_{6}(t), \quad t_{1}<t<t_{2} . \\ g(z / t) & \text { for } z_{6}(t) \leq z<z_{4}(t) \\ \Phi_{\infty} & \text { for } z \geq z_{4}(t)\end{cases}
$$

For the description of the solution beyond the time $t_{2}$ one sets $\Psi_{\infty}:=\left(0, \phi_{\max }\right)^{\mathrm{T}}, \Psi_{0}:=\Phi_{\mathrm{g}}\left(z^{*}, t^{*}\right), z^{*}:=z_{4}\left(t_{2}\right)$, $t^{*}:=t_{2}$ in order to handle the Riemann problem $R P\left(\Psi_{0}, \Psi_{\infty}\right)$, which can be solved analogously to $R P\left(\Phi_{0}, \Phi_{\infty}\right)$ with the simplification that the present Riemann problem is scalar. In the notation, all $\Phi$ need to be replaced by $\Psi$, and the map $g$, which is extracted from the parametrization of speed-state pairs, is now called $h$.

From $\left(z_{\mathrm{c} 2}, t_{2}\right)$, the discontinuities $z_{7}(t)=z_{\mathrm{c} 2}+\left(t-t_{2}\right) \lambda_{1}\left(\Psi_{0}^{*}\right), z_{8}(t)=z_{\mathrm{c} 2}+\left(t-t_{2}\right) \lambda_{1}\left(\Psi_{\infty}^{*}\right)$ and $z_{9}\left(t_{2}\right)=z_{\mathrm{c} 2}$ emerge. The trajectories $z_{5}(t)$ and $z_{7}(t)$ intersect at a time $t_{3}>t_{2}$, where we define $z_{\mathrm{c} 3}:=z_{5}\left(t_{3}\right)=z_{7}\left(t_{3}\right)$. The solution in the time interval $\left[t_{2}, t_{3}\right)$ becomes

$$
\Phi(z, t)= \begin{cases}0 & \text { for } z<z_{1}(t) \\ \Phi_{\mathrm{m}} & \text { for } z_{1}(t) \leq z<z_{5}(t), \\ \Phi_{\mathrm{g}}(z, t) & \text { for } z_{5}(t) \leq z<z_{7}(t), \quad t_{2}<t<t_{3} . \\ h\left(\left(z-z^{*}\right) /\left(t-t^{*}\right)\right) & \text { for } z_{7}(t) \leq z<z_{8}(t), \\ \Psi_{\infty} & \text { for } z_{8}(t) \leq z<z_{9}(t), \\ \Phi_{\infty} & \text { for } z \geq z_{9}(t)\end{cases}
$$

Meanwhile, no new zones emerge that require a special treatment of characteristics. From $\left(z_{\mathrm{c} 3}, t_{3}\right)$ there emerges the single curved kinematic shock defined by

$$
z_{10}\left(t_{3}\right)=z_{\mathrm{c} 3}, \quad \dot{z}_{10}=\sigma\left(h\left(\left(z_{10}(t)-z^{*}\right) /\left(t-t^{*}\right)\right), \Phi_{\mathrm{m}}\right), \quad t>t_{3},
$$

which intersects with $z_{1}(t)$ at a time $t_{4}$, where $z_{\mathrm{c} 4}=z_{1}\left(t_{4}\right)=z_{10}\left(t_{4}\right)$. The solution in the time interval $\left[t_{3}, t_{4}\right)$ becomes

$$
\Phi(z, t)= \begin{cases}0 & \text { for } z<z_{1}(t) \\ \Phi_{\mathrm{m}} & \text { for } z_{1}(t) \leq z<z_{10}(t), \\ h\left(\left(z-z^{*}\right) /\left(t-t^{*}\right)\right) & \text { for } z_{10}(t) \leq z<z_{8}(t), \quad t_{3}<t<t_{4} . \\ \Psi_{\infty} & \text { for } z_{8}(t) \leq z<z_{9}(t), \\ \Phi_{\infty} & \text { for } z \geq z_{9}(t)\end{cases}
$$

Finally, from $\left(z_{\mathrm{c} 4}, t_{4}\right)$ there emerges the single stationary shock $z_{11}\left(t_{4}\right)=z_{\mathrm{c} 4}$, so that for $t>t_{4}$ the solution becomes

$$
\Phi(z, t)= \begin{cases}0 & \text { for } z<z_{11} \\ \Psi_{\infty} & \text { for } z_{11} \leq z<z_{9}, \quad t>t_{4} . \\ \Phi_{\infty} & \text { for } z \geq z_{9}\end{cases}
$$

\subsection{Comparison with finite difference schemes}

The second example (Schneider et al. [47]) was solved by the Kurganov-Tadmor (KT) [30] finite difference scheme in [10]. The KT scheme includes measures to reduce numerical diffusivity. The fan width of Riemann problems at cell boundaries 
(a)

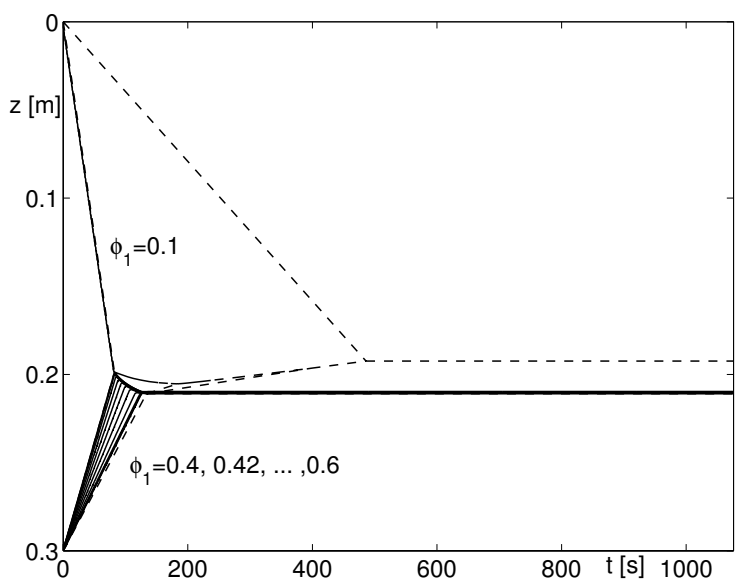

(c)

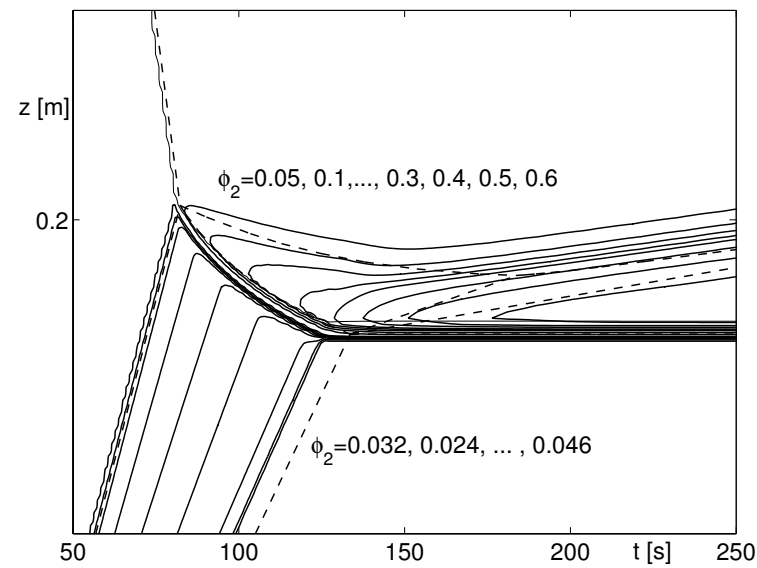

(b)

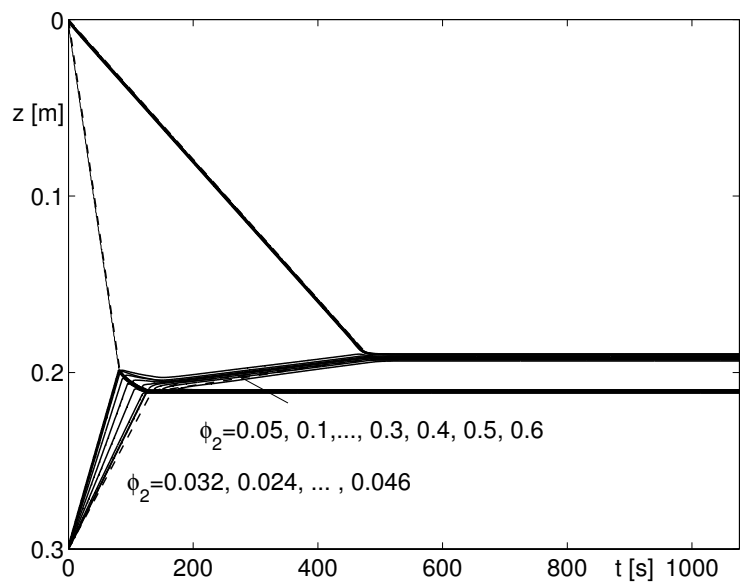

Fig. 15 Example 2: Contour lines of $\phi_{1}$ (a) and $\phi_{2}(\mathrm{~b}, \mathrm{c})$. The dashed contours describe the global solution as in Fig. 14.

(a) $t_{1}=T / 10$

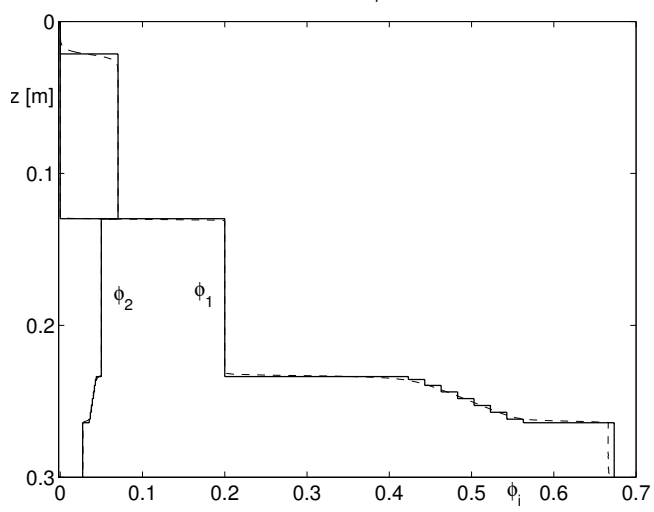

(b) $t_{2}=T / 5$

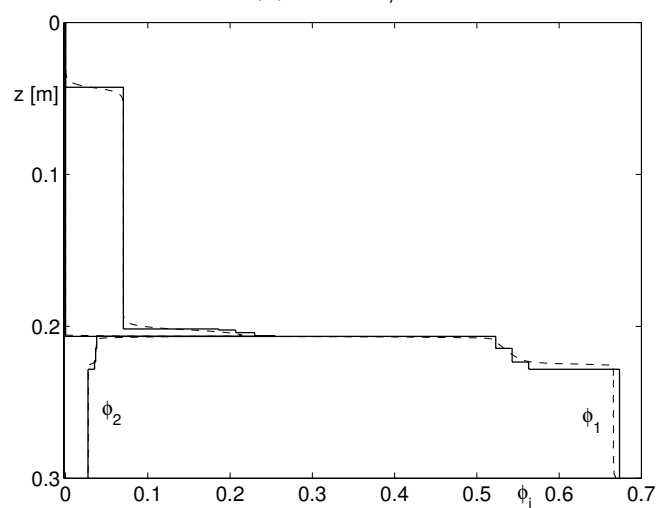

Fig. 16 Example 2: Comparison of profiles of $\phi_{1}$ and $\phi_{2}$, generated by front tracking (solid) and finite differences (dashed), $T=1076$. 
is estimated by the spectral radius of the Jacobian of the flux function. Nevertheless, if compared with the fronts obtained by the front tracking algorithm, the contour plots of those simulations still exhibit numerical diffusivity. The contour lines or profiles generated by finite difference schemes are sometimes ambiguous, since it may not be obvious whether a smooth concentration transition has a physical interpretation or is caused by numerical diffusivity.

For Figs. 15 and 16, where contours of small and large particle concentration and the concentration profiles at different times are plotted, the KT scheme [30] is used with discretization $J=500$ and a first-order reconstruction by a minmod limiter. Even though the KT scheme is quite accurate due to the high resolution, the numerical smearing makes it difficult to follow the process without ambiguity in the interpretation. For example, the zoomed plot of Fig. 15 (b) indicates that a second, upper sediment layer builds up on the first, but not how its creation may be explained in more detail. The generation of the centered rarefaction wave above the lower sediment and its interaction with the upper interfaces towards the buildup of the upper sediment have now been made visible.

For a single Riemann problem, a direct solution in the phase space is superior to any finite difference scheme that involves numerical diffusivity. In a phase-space analysis, the accuracy can be controlled rigorously. If one wants to use a front tracking code to solve general initial value problems, Riemann problems have to be solved each time when fronts collide. The complexity depends on the initial data. For oscillating initial data, which are approximated by piecewise constant initial data, numerous front interactions emerge, whereas for finite difference schemes the grid and thus the computational effort remains the same when the initial data are more complicated. For the initial datum (2.8) describing batch settling, the front tracking method is superior in the sense that it provides a resolution which has not been attained by finite difference schemes and thus gives detailed insights in the solution structure.

Acknowledgements We acknowledge support by Conicyt (Chile) through Fondecyt project 1050728 and Fondap in Applied Mathematics (project 15000001).

\section{References}

[1] N. Andrianov and G. Warnecke, The Riemann problem for the Baer-Nunziato two-phase flow model, J. Comput. Phys. 195, 434-464 (2004).

[2] F. Ancona and A. Marson, A note on the Riemann problem for general $n \times n$ conservation laws, J. Math. Anal. Appl. 260, 279-293 (2001).

[3] S. Benzoni-Gavage and R. Colombo, An n-populations model for traffic flow, Eur. J. Appl. Math. 14, 587-612 (2003).

[4] S. Berres, R. Bürger, and H. Frid, Neumann problems for quasi-linear parabolic systems modelling polydisperse suspensions, SIAM J. Math. Anal. 38, 557-573 (2006).

[5] S. Berres, R. Bürger, K. H. Karlsen, and E. M. Tory, Strongly degenerate parabolic-hyperbolic systems modeling polydisperse sedimentation with compression, SIAM J. Appl. Math. 64, 41-80 (2003).

[6] S. Bianchini, On the Riemann problem for non-conservative hyperbolic systems, Arch. Rational Mech. Anal. 166, 1-26 (2003).

[7] S. Bianchini and A. Bressan, Vanishing viscosity solutions of nonlinear hyperbolic systems, Ann. of Math. (2) 161, 223-342 (2005).

[8] A. Bressan, Hyperbolic Systems of Conservation Laws (Oxford University Press, Oxford, UK, 2000).

[9] R. Bürger, F. Concha, K.-K. Fjelde, and K. H. Karlsen, Numerical simulation of the settling of polydisperse suspensions of spheres, Powder Technol. 113, 30-54 (2000).

[10] R. Bürger, K.-K. Fjelde, K. Höfler, and K. H. Karlsen, Central difference solutions of the kinematic model of settling of polydisperse suspensions and three-dimensional particle-scale simulations, J. Eng. Math. 41, 167-187 (2001).

[11] R. Bürger, K. H. Karlsen, E. M. Tory, and W. L. Wendland, Model equations and instability regions for the sedimentation of polydisperse suspensions of spheres, ZAMM Z. Angew. Math. Mech. 82, 699-722 (2002).

[12] R. Bürger and A. Kozakevicius, Adaptive multiresolution WENO schemes for multi-species kinematic flow models, J. Comput. Phys. 224, 1190-1222 (2007).

[13] M. C. Bustos and F. Concha, Settling velocities of particulate systems: 10. A numerical method for solving Kynch sedimentation processes, Int. J. Mineral Process. 57, 185-203 (1999).

[14] M. C. Bustos, F. Concha, R. Bürger, and E. M. Tory, Sedimentation and Thickening: Phenomenological Foundation and Mathematical Theory (Kluwer Academic Publishers, Dordrecht, The Netherlands, 1999).

[15] C. M. Dafermos, Polygonal approximations of solutions of the initial value problem for a conservation law, J. Math. Anal. Appl. 38, 33-41 (1972).

[16] C. M. Dafermos, Hyperbolic Conservation Laws in Continuum Physics, Second Ed. (Springer-Verlag, Berlin, 2005).

[17] A. H. Falls and W.M. Schulte, Theory of three component, three phase displacement in porous media, SPE Reserv. Eng. 7, 377-384 (1992).

[18] A. H. Falls and W.M. Schulte, Features of three component, three phase displacement in porous media, SPE Reserv. Eng. 7, 426-432 (1992).

[19] E. Fried and B.C. Roy, Gravity-induced segregation of cohesionless granular mixture. In: K. Hutter and N. Kirchner (eds.), Dynamic Response of Granular and Porous Materials under Large and Catastrophic Deformations (Springer-Verlag, Berlin 2003, pp. 393-421). 
[20] V. Haugse, K. H. Karlsen, K.-A. Lie, and J. R. Natvig, Numerical solution of the polymer system by front tracking, Transp. Porous Media 44, 63-83 (2001).

[21] H. Holden, L. Holden, and R. A. Høegh-Krohn, A numerical method for first order nonlinear scalar conservation laws in one dimension, Comput. Math. Applic. 15, 595-602 (1988).

[22] H. Holden and N. H. Risebro, Front Tracking for Hyperbolic Conservation Laws (Springer-Verlag, New York, 2002).

[23] E. Isaacson, D. Marchesin, B. Plohr, and B. Temple, The Riemann problem near a hyperbolic singularity: the classification of solutions of quadratic Riemann problems I., SIAM J. Appl. Math. 48, 1009-1032 (1988).

[24] E. Isaacson and B. Temple, The Riemann problem near a hyperbolic singularity. II, III, SIAM J. Appl. Math. 48 1287-1301, 1302-1318 (1988).

[25] R. Juanes and K.-A. Lie, Numerical modeling of multiphase first-contact miscible flows. Part 1. Analytical Riemann solver, Transp. Porous Media 67, 375-393 (2007).

[26] R. Juanes and K.-A. Lie, Numerical modeling of multiphase first-contact miscible flows. Part 2. Front-tracking/streamline simulation, Transp. Porous Media, to appear.

[27] R. Juanes and T. W. Patzek, Three-phase displacement theory: An improved description of relative permeabilities, SPE J. 9, 302-313 (2004).

[28] R. Juanes and T. W. Patzek, Analytical solution to the Riemann problem of three-phase flow in porous media, Transp. Porous Media 55, 47-70 (2004).

[29] B. L. Keyfitz and H. C. Kranzer, A viscosity approximation to a system of conservation laws with no classical Riemann solution. In: Nonlinear Hyperbolic Problems (Bordeaux, 1988), Lecture Notes in Math. 1402 (Springer-Verlag, Berlin 1989), pp. $185-197$.

[30] A. Kurganov and E. Tadmor, New high-resolution central schemes for nonlinear conservation laws and convection-diffusion equations, J. Comput. Phys. 160, 214-282 (2000).

[31] G. J. Kynch, A theory of sedimentation, Trans. Farad. Soc. 48, 166-176 (1952).

[32] P. D. Lax, Hyperbolic systems of conservation laws II, Comm. Pure Appl. Math. 10, 537-566 (1957).

[33] P.D. Lax, Hyperbolic Systems of Conservation Laws and the Mathematical Theory of Shock Waves (SIAM, Philadelphia, PA, 1973).

[34] R. J. LeVeque, Numerical Methods for Conservation Laws, Second Ed. (Birkhäuser Verlag, Basel, 1992).

[35] K.-A. Lie and R. Juanes, A front-tracking method for the simulation of three-phase flow in porous media, Comput. Geosci. 9 , 29-59 (2005).

[36] T. P. Liu, The Riemann problem for general $2 \times 2$ conservation laws, Trans. Amer. Math. Soc. 199, 89-112 (1974).

[37] T. P. Liu, The entropy condition and the admissibility of shocks, J. Math. Anal. Appl. 53, 78-88 (1976).

[38] M. J. Lockett and K. S. Bassoon, Sedimentation of binary particle mixtures, Powder Technol. 24, 1-7 (1979).

[39] A. Majda and R. L. Pego, Stable viscosity matrices for systems of conservation laws, J. Diff. Eqns. 56, 229-262 (1985).

[40] D. Marchesin and B. Plohr, Wave structure in WAG recovery, SPE paper 56480.

[41] J. H. Masliyah, Hindered settling in a multiple-species particle system, Chem. Eng. Sci. 34, 1166-1168 (1979).

[42] R. Menikoff and B. J. Plohr, The Riemann problem for fluid flow of real materials, Rev. Mod. Phys. 61, 75-130 (1989).

[43] S. Müller and A. Voß, The Riemann Problem for the Euler equations with nonconvex and nonsmooth equation of state: Construction of wave curves, SIAM J. Sci. Comp. 28, 651-681 (2006).

[44] N. H. Risebro, A front tracking alternative to the random choice method, Proc. Amer. Math. Soc. 117, 1125-1139 (1993).

[45] N. H. Risebro and A. Tveito, Front tracking applied to a nonstrictly hyperbolic system of conservation laws, SIAM J. Sci. Stat. Comput. 12, 1401-1419 (1991).

[46] F. Rosso and G. Sona, Gravity-driven separation of oil-water dispersions, Adv. Math. Sci. Appl. 11, 127-151 (2001).

[47] W. Schneider, G. Anestis, and U. Schaflinger, Sediment composition due to settling of particles of different sizes, Int. J. Multiphase Flow 11, 419-323 (1985).

[48] P. T. Shannon, E. Stroupe, and E. M. Tory, Batch and continuous thickening, Ind. Eng. Chem. Fund. 2, $203-211$ (1963).

[49] M. Torrilhon, Uniqueness conditions for Riemann problems of ideal magnetohydrodynamics, J. Plasma Phys. 69, $253-276$ (2003).

[50] B. Wendroff, An analysis of front tracking for chromatography, Acta Appl. Math. 30, 265-285 (1993).

[51] G. C. K. Wong and S. C. Wong, A multi-class traffic flow model - an extension of LWR model with heterogeneous drivers, Transp. Res. A 36, 827-841 (2002).

[52] A. Zeidan, A. Rohani, and A. Bassi, Dynamic and steady-state sedimentation of polydisperse suspension and prediction of outlets particle-size distribution, Chem. Eng. Sci. 59, 2619-2632 (2004).

[53] A. Zeidan, S. Rohani, A. Bassi, and P. Whiting, Review and comparison of solids settling velocity models, Rev. Chem. Eng. 19, 473-530 (2003). 